\title{
RESTRICTING UNIPOTENT CHARACTERS IN FINITE SYMPLECTIC GROUPS
}

\author{
JIANBEI AN AND GERHARD HISS
}

\begin{abstract}
We compute the irreducible constituents of the restrictions of all unipotent characters of the groups $\operatorname{Sp}_{4}(q)$ and $\operatorname{Sp}_{6}(q)$ and odd $q$ to their maximal parabolic subgroups stabilizing a line. It turns out that these restrictions are multiplicity free.

We also obtain general information about the restrictions of Harish-Chandra induced characters.
\end{abstract}

\section{INTRODUCTION}

This is a sequel to our paper [1], where we investigated the restriction of the Steinberg character of a finite symplectic group $G$ over a field of odd characteristic to the maximal parabolic subgroup $P$ of $G$ stabilizing a line. We determined the irreducible constituents of this restriction up to the constituents in the product of the Steinberg character of a Levi subgroup $L$ of $P$ with a Weil character of $L$. This information was sufficient to get a complete answer for $G=\operatorname{Sp}_{4}(q)$ and $\operatorname{Sp}_{6}(q)$.

The constituents of the product of the Steinberg character with a Weil character were computed in [6] for all finite symplectic groups, thus completing the work of [1]. It turned out that the restriction of the Steinberg character of $G$ to $P$ is multiplicity free.

This paper is the first step in generalizing this result to all unipotent characters. The information we obtain is sufficient to determine the irreducible constituents of the unipotent characters for $G=\operatorname{Sp}_{4}(q)$ and $\operatorname{Sp}_{6}(q)$, and again, all these restrictions are multiplicity free.

There is not much evidence that this pattern should persist for $G=$ $\operatorname{Sp}_{2 m}(q)$ for $m>2$, but we do conjecture that there is an upper bound, only depending on the Lie rank of $G$ but not on $q$, for the composition multiplicities in the restrictions of the unipotent characters of $G$ to $P$.

Our motivation for this work is the $\ell$-modular representation theory of $G$. Suppose that there exists such a bound $M$ as above. Let $\chi$

2000 Mathematics Subject Classification. 20C33 (20C15 20G40).

Key words and phrases. Symplectic Groups, Unipotent Characters, Parabolic Subgroup. 
be a unipotent character of $G$. Then it follows from a standard argument, that the $\ell$-modular decomposition numbers $d_{\chi, \varphi}$ are bounded from above by $M c$, where $c$ is the largest diagonal entry in the $\ell$ modular Cartan matrix of $P$. Since by Clifford theory the $\ell$-modular representation theory of $P$ can be reduced to that of $L$ and to the parabolic subgroup of $L$ analogous to $P$, the above bound is a basis for induction. For example, it has been used in [1] to prove Donovan's conjecture for $\operatorname{Sp}_{6}(q)$ if $\ell>3$.

Our general results, true for all symplectic groups $G$, are based on Mackey's theorem. Namely, we do not restrict individual unipotent characters of $G$ to $P$, but characters obtained from Harish-Chandra induction from $L$. That is, we start with an irreducible character $\sigma$ of $L$, view it as a character of $P$ via inflation, and consider the character $\chi$ of $G$ obtained by inducing $\sigma$ from $P$ to $G$. One part of the restriction of $\chi$ to $P$ is determined recursively, the other part only modulo the constituents of the product of $\sigma$ with a Weil character of $L$. As an intermediate result we compute all these products in $\operatorname{Sp}_{4}(q)$.

This method does not give information about the restrictions of cuspidal unipotent characters, of course. However, the restriction of the cuspidal unipotent character of $\operatorname{Sp}_{4}(q)$ to $P$ can be obtained by other means, and $\mathrm{Sp}_{6}(q)$ does not have any cuspidal unipotent character. Our results show that, in order to establish the existence of a bound $M$ as conjectured above, it would suffice to restrict cuspidal unipotent characters and to bound the multiplicities in the products of unipotent characters with the Weil characters.

\section{Notation AND RECOLLECTIONS}

In this section we fix some notation and recall some of the results of [1], taking the opportunity to correct some inaccuracies of that paper.

Throughout our paper, we write $G:=G_{n}:=\operatorname{Sp}_{n}(q)$ for a nonnegative even integer $n=2 m$ and an odd prime power $q$. We adopt the convention that $G_{0}=\operatorname{Sp}_{0}(q)$ is the trivial group. To be specific, we always take $G$ to be the matrix group

$$
G:=G_{n}:=\operatorname{Sp}_{n}(q):=\left\{\mathbf{x} \in \operatorname{GL}_{n}(q) \mid \mathbf{x}^{t} \tilde{J}_{n} \mathbf{x}=\tilde{J}_{n}\right\} .
$$

(As in [1], we usually denote matrices by boldface letters.) With respect to the standard basis of the column vector space $\mathbf{F}_{q}^{n}$, the Gram matrix of our symplectic form defining $G$ has shape

$$
\tilde{J}_{n}:=\left[\begin{array}{cc}
0 & J_{m} \\
-J_{m} & 0
\end{array}\right],
$$


where $J_{m}$ denotes the $(m \times m)$-matrix with 1 s along the anti-diagonal and 0 s elsewhere.

The following special matrices and subgroups are relevant for our paper. Usually the index $n$ on the symbol for a subgroup indicates the format of the matrices of its elements. Assume $n \geq 2$ for the remainder of this section. For $\mathbf{x} \in \operatorname{Sp}_{n-2}(q)$ and $a \in \mathbb{F}_{q}^{*}$, we put

$$
\mathbf{s}_{n}(\mathbf{x}, a):=\left[\begin{array}{ccc}
a & 0 & 0 \\
0 & \mathbf{x} & 0 \\
0 & 0 & a^{-1}
\end{array}\right]
$$

and

$$
L:=L_{n}:=\left\{\mathbf{s}_{n}(\mathbf{x}, a) \mid \mathbf{x} \in \operatorname{Sp}_{n-2}(q), a \in \mathbb{F}_{q}^{*}\right\} .
$$

We write $L_{n}^{\prime}$ for the subgroup of $L_{n}$ consisting of the matrices $\mathbf{s}_{n}(\mathbf{x}, a)$ of (1) with $a=1$. Then $L_{n}^{\prime} \cong \operatorname{Sp}_{n-2}(q)$ and except for $n=4$ and $q=3, L_{n}^{\prime}$ is the commutator subgroup of $L_{n}$. We have the factorization $L_{n}=A \times L_{n}^{\prime}$, where

$$
A:=A_{n}:=\left\{\left[\begin{array}{ccc}
a & 0 & 0 \\
0 & I_{n-2} & 0 \\
0 & 0 & a^{-1}
\end{array}\right] \mid a \in \mathbb{F}_{q}^{*}\right\} .
$$

(Here and in the following, we write $I_{n}$ for the $(n \times n)$-identity matrix.) Occasionally we identify $G_{n-2}=\operatorname{Sp}_{n-2}(q)$ with the subgroup $L_{n}^{\prime}$ of $G_{n}$.

Next, for $\mathbf{v} \in \mathbb{F}_{q}^{n-2}$ and $z \in \mathbb{F}_{q}$ we put

$$
\mathbf{u}_{n}(\mathbf{v}, z):=\left[\begin{array}{ccc}
1 & \mathbf{v}^{t} \tilde{J}_{n-2} & z \\
0 & I_{n-2} & \mathbf{v} \\
0 & 0 & 1
\end{array}\right]
$$

and

$$
U:=U_{n}:=\left\{\mathbf{u}_{n}(\mathbf{v}, z) \mid \mathbf{v} \in \mathbb{F}_{q}^{n-2}, z \in \mathbb{F}_{q}\right\}
$$

Then $P:=P_{n}:=U_{n} L_{n}$ is a maximal parabolic subgroup of $G$ fixing the line $\left\langle[1,0, \ldots, 0]^{t}\right\rangle$. The group $U$ is the unipotent radical of $P$ and $L$ is its Levi complement. The center $Z(U)$ of $U$ consists of the matrices $\mathbf{u}_{n}(0, z), z \in \mathbb{F}_{q}$, the quotient $U / Z(U)$ is isomorphic to $\mathbb{F}_{q}^{n-2}$, and the action of $L_{n}^{\prime}$ on $U / Z(U)$ by conjugation is equivalent to the natural action of $\operatorname{Sp}_{n-2}(q)$ on $\mathbb{F}_{q}^{n-2}$.

The ordinary irreducible characters of $P$ can be classified into three types:

Type 1: Characters with $U$ in their kernel.

Type 2: Characters with $Z(U)$ but not $U$ in their kernel.

Type 3: Characters with $Z(U)$ not in their kernel. 
Note that for $n=2$ there are no characters of Type 2 (since $Z(U)=U$ in this case). We now recall the parametrization and construction of the characters of $P$ from [1, Section 2.2].

Characters of Type 1 are parametrized by $\operatorname{Irr}(L)$. The character of Type 1 corresponding to $\sigma \in \operatorname{Irr}(L)$ is denoted by ${ }^{1} \psi_{\sigma}$ and defined by

$$
{ }^{1} \psi_{\sigma}=\operatorname{Infl}_{L}^{P}(\sigma) \text {. }
$$

We have ${ }^{1} \psi_{\sigma}(1)=\sigma(1)$.

Characters of Type 2 are parametrized by $\operatorname{Irr}\left(P_{n-2}\right)$. The character of Type 2 corresponding to $\mu \in \operatorname{Irr}\left(P_{n-2}\right)$ is denoted by ${ }^{2} \psi_{\mu}$ and defined as follows. Suppose that $n \geq 4$ and let $\zeta$ be a nontrivial irreducible complex character of the additive group of $\mathbb{F}_{q}$. Define $\lambda \in \operatorname{Irr}(U)$ by $\lambda\left(\mathbf{u}_{n}(\mathbf{v}, z)\right):=\zeta\left(v_{n-2}\right)$, where $\mathbf{v}=\left[v_{1}, \ldots, v_{n-2}\right]^{t} \in \mathbb{F}_{q}^{n-2}$. Let $T_{n}:=$ $T_{P}(\lambda)$ be the inertia subgroup of $\lambda$ in $P$. Then $T_{n}=U \tilde{P}_{n-2}$ with a subgroup $\tilde{P}_{n-2}$ of $L$ satisfying $A \times \tilde{P}_{n-2}=A \times P_{n-2}$. (Contrary to what we have written in $[1$, p. 254 , penultimate line], we identify $P_{n-2} \leq G_{n-2}$ with the subgroup $\left\{\mathbf{s}_{n}(\mathbf{x}, 1) \mid \mathbf{x} \in P_{n-2}\right\} \leq L \leq G$.) For the precise form of $\tilde{P}_{n-2}$ see $[1,2.3 .2]$. Let $\hat{\lambda}$ be the extension of $\lambda$ to $T_{n}$ such that $\operatorname{Res}_{\tilde{P}_{n-2}}^{T_{n}}(\hat{\lambda})=1_{\tilde{P}_{n-2}}$. For $\mu \in \operatorname{Irr}\left(P_{n-2}\right)$ put $\tilde{\mu}:=$ $\operatorname{Res}_{\tilde{P}_{n-2} \times P_{n-2}}^{A}\left(1_{A} \otimes \mu\right) \in \operatorname{Irr}\left(\tilde{P}_{n-2}\right)$. Then

$$
{ }^{2} \psi_{\mu}:=\operatorname{Ind}_{T_{n}}^{P_{n}}\left(\hat{\lambda} \cdot \operatorname{Infl}_{\tilde{P}_{n-2}}^{T_{n}}(\tilde{\mu})\right) .
$$

We have ${ }^{2} \psi_{\mu}(1)=\left(q^{n-2}-1\right) \mu(1)$.

Characters of Type 3 are parametrized by $\operatorname{Irr}\left(L^{\prime}\right)$ in such a way that each $\vartheta \in \operatorname{Irr}\left(L^{\prime}\right)$ parametrizes four characters of $G$, denoted by ${ }^{3} \psi_{\vartheta}^{i, \varepsilon}$ with $i \in\{1,2\}$ and $\varepsilon \in\{+,-\}$. These are constructed as follows. To have a consistent choice for all the symplectic groups we are considering, we fix two non-trivial irreducible complex characters $\zeta_{1}$ and $\zeta_{2}$ of the additive group of $\mathbb{F}_{q}$, such that $\zeta_{1}$ and $\zeta_{2}$ are not conjugate under the group of squares of $\mathbb{F}_{q}^{*}$. We view $\zeta_{1}$ and $\zeta_{2}$ as irreducible characters of $Z(U)$ via the isomorphism $z \mapsto \mathbf{u}_{n}(0, z)$. Then $\zeta_{1}$ and $\zeta_{2}$ are representatives of the two orbits of $A$ on $\operatorname{Irr}(U) \backslash\left\{1_{U}\right\}$. There are unique irreducible characters $\rho_{i}$ of $U$ with $\rho_{i}(1)=q^{m-1}$ (recall that $n=2 m$ ) such that $\operatorname{Res}_{Z(U)}^{U}\left(\rho_{i}\right)=q^{m-1} \zeta_{i}, i=1,2$. The inertia subgroup of the $\rho_{i}$ equals $P^{\prime} Z=P^{\prime} \times Z$ with $Z=Z(G)$ and $P^{\prime}=U L^{\prime}$. Moreoever, the $\rho_{i}$ extend to characters $\hat{\rho}_{i}$ of $P^{\prime}$, and these extensions are unique except in the case $n=4$ and $q=3$, where we choose the extensions as in [5, Theorem 2.4]. The trivial extensions of the $\hat{\rho}_{i}$ to $P^{\prime} Z$ are denoted by the same symbols. Each irreducible character $\vartheta$ of $L^{\prime}$ has two extensions to $L^{\prime} Z=L^{\prime} \times Z$, namely $\vartheta \cdot 1_{Z}^{\varepsilon}$ with the sign $\varepsilon \in\{+,-\}$, where $1_{Z}^{+}$ 
and $1_{Z}^{-}$denote the trivial and non-trivial irreducible characters of $Z$, respectively. For each such $\vartheta$ we put

$$
{ }^{3} \psi_{\vartheta}^{i, \varepsilon}:=\operatorname{Ind}_{P^{\prime} Z}^{P}\left(\hat{\rho}_{i} \cdot \operatorname{Infl}_{L^{\prime} Z}^{P^{\prime} Z}\left(\vartheta \cdot 1_{Z}^{\varepsilon}\right)\right),
$$

$i=1,2$ and $\varepsilon \in\{+,-\}$. We have ${ }^{3} \psi_{\vartheta}^{i, \varepsilon}(1)=q^{m-1}(q-1) \vartheta(1) / 2$.

It is convenient to expand the parametrization of the irreducible characters of $P$ of a given type to all characters of this type. This is achieved by a linear extension of the parametrizing map. Thus, for example, if $\sigma=\sum n_{i} \sigma_{i}$ is a character of $L$, where the $n_{i}$ are positive integers and $\sigma_{i} \in \operatorname{Irr}(L)$, then ${ }^{1} \psi_{\sigma}$ denotes the Type 1 character $\sum n_{i}{ }^{1} \psi_{\sigma_{i}}$ of $P$.

Finally, we introduce some pieces of character theoretic notation. Let $H$ be a finite group. Character always means complex character. If $\chi$ is a character of $H$, we write $\bar{\chi}$ for the complex conjugate of $\chi$. The trivial character of $H$ is denoted by $1_{H}$. If $H$ has a unique normal subgroup of index 2 , we write $1_{H}^{-}$for the unique linear character of $H$ of order 2. Given two characters $\chi$ and $\psi$ of $H$, we say that $\psi$ is a subcharacter of $\chi$ if $\chi-\psi$ is a character.

\section{A FiRST APPROACH FOR RESTRICTING UNIPOTENT CHARACTERS}

It will be useful for our explicit calculations in the cases $m=2$ and $m=3$ to discuss a general approach for obtaining information about composition multiplicities of certain unipotent characters restricted to $P$. Throughout this section, we fix an irreducible character $\sigma$ of $L^{\prime}$, extended trivially to a character, also denoted by $\sigma$, of $L=A \times L^{\prime}$. We write $R_{L}^{G}(\sigma)$ for the Harish-Chandra induced character, i.e., $R_{L}^{G}(\sigma)=\operatorname{Ind}_{P}^{G}(\sigma)$, where $\sigma$ is viewed as a character of $P$ via inflation. We are interested in $\operatorname{Res}_{P}^{G}\left(R_{L}^{G}(\sigma)\right)$. Every unipotent character of $G$, except a cuspidal one, is a constituent in some such $R_{L}^{G}(\sigma)$. Thus our approach is restricted to the non-cuspidal unipotent characters. Moreoever, we do not obtain the restriction of a single irreducible character, but only of the sum of certain irreducibles. Nevertheless, this method has turned out to be useful in our specific computations.

The Weyl group $W$ of $G$ is of type $C_{m}$. Let us denote the standard reflections of $W$ by $S:=\left\{s_{1}, s_{2}, \ldots, s_{m}\right\}$, where we choose notation in such a way that $s_{2}, \ldots, s_{m}$ are conjugate in $W$ and $s_{1}$ is not conjugate to any of $s_{2}, \ldots, s_{m}$. (Alternatively, $s_{1}$ is the reflection along a long root and $s_{2}, \ldots, s_{m}$ are reflections along short roots.) Putting $J:=$ $S \backslash\left\{s_{m}\right\}$, then $P$ is the standard parabolic subgroup $P_{J}$ and $L=L_{J}$ is its standard Levi subgroup. 
Mackey's theorem yields

$$
\operatorname{Res}_{P}^{G}\left(R_{L}^{G}(\sigma)\right)=\sum_{d \in D_{J, J}} \operatorname{Ind}_{d P \cap P}^{P}\left(\operatorname{Res}_{d P \cap P}^{d_{P}}\left({ }^{d} \sigma\right)\right),
$$

where $D_{J, J}$ is the set of distinguished double coset representatives for the $W_{J}-W_{J}$-double coset of $W$ (see $[2$, Section 2.7]). (We write, unambiguously, ${ }^{d} P$ and ${ }^{d} \sigma$ for ${ }^{d} P$ and ${ }^{d_{\sigma}}$, respectively, where $\dot{d}$ is a suitable inverse image of $d$ in $G$ as in [2, Section 2.8].)

First we collect some known facts about $D_{J, J}$ and the groups ${ }^{d} P \cap P$ for $d \in D_{J, J}$.

Lemma 3.1. Assume $m \geq 2$, let $J=\left\{s_{1}, \ldots, s_{m-1}\right\}$ and put $s:=s_{m}$ and $t:=s_{m} s_{m-1} \cdots s_{2} s_{1} s_{2} \cdots s_{m-1} s_{m}$.

(a) $D_{J, J}=\{1, s, t\}$.

(b) ${ }^{t} P \cap P=L$.

(c) Put $K:={ }^{s} J \cap J$. Then $K=\left\{s_{1}, \ldots, s_{m-2}\right\}$ and

$$
{ }^{s} P \cap P=\left({ }^{s} U \cap U\right)\left({ }^{s} L \cap U\right)\left({ }^{s} U \cap L\right) L_{K} .
$$

Moreoever, $Q_{K}:=\left({ }^{s} U \cap L\right) L_{K}$ is the standard parabolic subgroup of $L$ corresponding to $K$, and ${ }^{s} U \cap L$ is the unipotent radical of $Q_{K}$.

(d) We have $Z(U) \leq{ }^{s} L \cap U$ and ${ }^{s} U \cap U$ has order $q$.

Put $R:=\left({ }^{s} U \cap U\right)\left({ }^{s} L \cap U\right)$. Then ${ }^{s} P \cap P=R Q_{K}$. We have $R=$ $\left\{\mathbf{u}_{n}(\mathbf{v}, z) \mid \mathbf{v}=\left(v_{1}, \ldots, v_{n-2}\right) \in \mathbb{F}_{q}^{n-2}, v_{n-2}=0, z \in \mathbb{F}_{q}\right\}$. In particular, $R$ has index $q$ in $U$ and so $R Q_{K}$ has index $q$ in $P_{K}=U Q_{K}$. Finally, $Q_{K}=A \times P_{n-2}$.

Proof. (a) For $1 \leq j \leq m$ put $r_{j}:=s_{j} \cdots s_{m-1} s_{m}$. Let $D_{J}$ denote the set of distinguished left coset representatives of the parabolic subgroup $W_{J}$ in $W$. Then

$$
D_{J}=\left\{1, t, r_{j}, r_{j}^{-1} t \mid 2 \leq j \leq m\right\} .
$$

The result now follows from $D_{J, J}=D_{J}^{-1} \cap D_{J}$ (see [2, Section 2.7]).

(b) This is easy to check by a direct computation.

(c) Since $s$ commutes with each of $s_{1}, \ldots, s_{m-2}$, but not with $s_{m-1}$, it is clear that $K$ is as claimed. The remaining statements are proved in $[2,2.8]$.

(d) This is clear.

It will perhaps help the reader to follow the somewhat technical arguments in Theorem 3.3 below, if we give the relevant structures of Lemma 3.1 in terms of matrices. First we describe inverse images of $s$ 
and $t$ in $G$ (denoted by the same letters). We may take

$$
s=\left[\begin{array}{ccc}
J_{2} & 0 & 0 \\
0 & I_{n-4} & 0 \\
0 & 0 & J_{2}
\end{array}\right]
$$

and

$$
t=\left[\begin{array}{ccc}
0 & 0 & 1 \\
0 & I_{n-2} & 0 \\
-1 & 0 & 0
\end{array}\right]
$$

Then

$$
{ }^{s} U \cap U=\left\{\left[\begin{array}{ccc}
I_{2} & 0 & a I_{2} \\
0 & I_{n-4} & 0 \\
0 & 0 & I_{2}
\end{array}\right] \mid a \in \mathbb{F}_{q}\right\}
$$

and

$$
{ }^{s} U \cap L=\left\{\left[\begin{array}{ccc}
1 & 0 & 0 \\
0 & \mathbf{u}_{n-2}(\mathbf{v}, z) & 0 \\
0 & 0 & 1
\end{array}\right] \mid \mathbf{v} \in \mathbb{F}_{q}^{n-4}, z \in \mathbb{F}_{q}\right\} .
$$

Note that ${ }^{s} U \cap L$ was called $\tilde{U}_{n-2}$ in [1, 2.3.2], but here we will just call it $U_{n-2}$ (in accordance with our convention to view $G_{n-2}$ as a subgroup of $G$ ). Putting

$$
A_{n-2}:={ }^{s} A
$$

we have

$$
{ }^{s} L \cap L=L_{K}=A \times A_{n-2} \times L_{n-2}^{\prime}
$$

with

$$
L_{n-2}^{\prime}=\left\{\left[\begin{array}{ccc}
I_{2} & 0 & 0 \\
0 & \mathbf{x} & 0 \\
0 & 0 & I_{2}
\end{array}\right] \mid \mathbf{x} \in \operatorname{Sp}_{n-4}(q)\right\} \cong \operatorname{Sp}_{n-4}(q) .
$$

For the proof of the main theorem of this section we need the following technical lemma.

Lemma 3.2. For $n \geq 6$ put

$$
A_{n, n-2}:=\left\{\left[\begin{array}{ccc}
a I_{2} & 0 & 0 \\
0 & I_{n-4} & 0 \\
0 & 0 & a^{-1} I_{2}
\end{array}\right] \mid a \in \mathbb{F}_{q}^{*}\right\},
$$

$r:=s_{m-1}$, and $A_{n-4}:={ }^{r} A_{n-2}$. Furthermore, let

$$
Q_{K}^{\prime}:=A \times U_{n-2} \tilde{P}_{n-4} \leq Q_{K} .
$$

Then

$$
{ }^{r s}\left(R Q_{K^{\prime}}\right) \cap U \tilde{P}_{n-2}=\left({ }^{r} R\right) Y
$$


with

$Y=\left({ }^{r} U_{n-2} \cap U_{n-2}\right)\left({ }^{r} L_{n-2} \cap U_{n-2}\right)\left({ }^{r} U_{n-2} \cap L_{n-2}\right)\left(A_{n, n-2} \times A_{n-4} \times L_{n-4}^{\prime}\right)$.

In particular, $A \times Y=A \times\left({ }^{r} P_{n-2} \cap P_{n-2}\right)$.

Proof. We have

$$
\tilde{P}_{n-2}=A_{n, n-2} U_{n-2} L_{n-2}^{\prime}
$$

and

$$
Q_{K}^{\prime}=A U_{n-2} \tilde{P}_{n-4}=A U_{n-2}^{s r} A_{n, n-2} U_{n-4} L_{n-4}^{\prime}
$$

Hence

$$
{ }^{r s}\left(R Q_{K^{\prime}}\right)=\left({ }^{r} R\right) A_{n, n-2}{ }^{r}\left(A_{n-2} U_{n-2} U_{n-4} L_{n-4}^{\prime}\right),
$$

since ${ }^{s}\left(R U_{n-2}\right)=R U_{n-2}$. This easily implies that

$$
{ }^{r s}\left(R Q_{K^{\prime}}\right) \cap U \tilde{P}_{n-2}=\left({ }^{r} R\right) Y
$$

with

$$
\begin{aligned}
Y & :=A_{n, n-2} U_{n-2} L_{n-2}^{\prime} \cap A_{n, n-2}{ }^{r}\left(A_{n-2} U_{n-2} U_{n-4} L_{n-4}^{\prime}\right) \\
& =A_{n, n-2}\left[U_{n-2} L_{n-2}^{\prime} \cap{ }^{r}\left(A_{n-2} U_{n-2} U_{n-4} L_{n-4}^{\prime}\right)\right] .
\end{aligned}
$$

By Lemma 3.1(c), applied to $G_{n-2}$, we have

$$
\begin{gathered}
{ }^{r} P_{n-2} \cap P_{n-2}= \\
\left({ }^{r} U_{n-2} \cap U_{n-2}\right)\left({ }^{r} L_{n-2} \cap U_{n-2}\right)\left({ }^{r} U_{n-2} \cap L_{n-2}\right)\left(A_{n-2} \times A_{n-4} \times L_{n-4}^{\prime}\right) .
\end{gathered}
$$

Put

$$
Y^{\prime}:=\left({ }^{r} U_{n-2} \cap U_{n-2}\right)\left({ }^{r} L_{n-2} \cap U_{n-2}\right)\left({ }^{r} U_{n-2} \cap L_{n-2}\right)\left(A_{n-4} \times L_{n-4}^{\prime}\right),
$$

so that ${ }^{r} P_{n-2} \cap P_{n-2}=A_{n-2} Y^{\prime}$. Clearly,

$$
Y^{\prime} \leq U_{n-2} L_{n-2}^{\prime} \cap^{r}\left(A_{n-2} U_{n-2} U_{n-4} L_{n-4}^{\prime}\right),
$$

since ${ }^{r} L_{n-2} \cap U_{n-2}={ }^{r} U_{n-4}$.

On the other hand, $U_{n-2} L_{n-2}^{\prime}=P_{n-2}^{\prime} \leq P_{n-2}$, and so

$$
\begin{aligned}
U_{n-2} L_{n-2}^{\prime} \cap{ }^{r}\left(A_{n-2} U_{n-2} U_{n-4} L_{n-4}^{\prime}\right) & \leq P_{n-2}^{\prime} \cap{ }^{r} P_{n-2} \\
& =P_{n-2}^{\prime} \cap{ }^{r} P_{n-2} \cap P_{n-2} \\
& =P_{n-2}^{\prime} \cap A_{n-2} Y^{\prime}=Y^{\prime} .
\end{aligned}
$$

Thus $Y=A_{n, n-2} Y^{\prime}$ and the result follows.

We now come to the main result of this section. Recall that $P=P_{J}$ with $J=S \backslash\left\{s_{m}\right\}$ and that we are interested in $\operatorname{Res}_{P}^{G}\left(R_{L}^{G}(\sigma)\right)$ for an irreducible character $\sigma$ of $L$ with $A$ in its kernel. In view of (3) and Lemma 3.1, we have to determine

$$
\operatorname{Ind}_{L}^{P}\left(\operatorname{Res}_{L}^{t} P\left({ }^{t} \sigma\right)\right)
$$


and

$$
\operatorname{Ind}_{R Q_{K}}^{P}\left(\operatorname{Res}_{R Q_{K}}^{s}\left({ }^{s} \sigma\right)\right) .
$$

Since $R Q_{K}={ }^{s} P \cap P$ is $s$-invariant, we have $\operatorname{Res}_{R Q_{K}}^{s^{s}}\left({ }^{s} \sigma\right)={ }^{s}\left(\operatorname{Res}_{R Q_{K}}^{P}(\sigma)\right)$. Also, $\operatorname{Res}_{R Q_{K}}^{P}(\sigma)=\operatorname{Infl}_{P_{n-2}}^{R Q_{K}}\left(\operatorname{Res}_{P_{n-2}}^{L^{\prime}}(\sigma)\right)$. Thus $\operatorname{Ind}_{R Q_{K}}^{P}\left(\operatorname{Res}_{R Q_{K}}^{s_{P}}\left({ }^{s} \sigma\right)\right)$ is a sum of characters of the form $\operatorname{Ind}_{R Q_{K}}^{P}\left({ }^{s} \nu\right)$, where $\nu$ is an irreducible character of $P_{n-2}$, viewed as a character of $R Q_{K}=R\left(A \times P_{n-2}\right)$ via inflation. The answer depends on the type of $\nu$.

Theorem 3.3. Assume that $n \geq 4$. Let $\sigma$ be an irreducible character of $L$ with $A$ in its kernel. Then the following statements hold.

(a) $\operatorname{Res}_{L}^{t^{t}}\left({ }^{t} \sigma\right)=\sigma$. In particular,

$$
\operatorname{Ind}_{t P \cap P}^{P}\left(\operatorname{Res}_{t P \cap P}^{t_{P}}\left({ }^{t} \sigma\right)\right)=\operatorname{Ind}_{L}^{P}(\sigma) .
$$

(b) Let $\nu$ be an irreducible character of $P_{n-2}$ of Type 1 . We view $\nu$ as an irreducible character of $L_{K}=A \times L_{n-2}=A \times A_{n-2} \times L_{n-2}^{\prime}$, and also as an irreducible character of $R Q_{K}$ via inflation. Then

$$
\operatorname{Ind}_{R Q_{K}}^{P}\left({ }^{s} \nu\right)={ }^{1} \psi_{\Sigma}+{ }^{2} \psi_{\nu}
$$

where $\Sigma=R_{L_{K}}^{L}\left({ }^{s} \nu\right)$.

(c) Suppose that $n \geq 6$ and let $\nu$ be an irreducible character of $P_{n-2}$ of Type 2. Suppose that $\nu={ }^{2} \psi_{\nu_{0}}$, with $\nu_{0} \in \operatorname{Irr}\left(P_{n-4}\right)$. Then

$$
\operatorname{Ind}_{R Q_{K}}^{P}\left({ }^{s} \nu\right)={ }^{2} \psi_{\Sigma}
$$

with $\Sigma=\operatorname{Ind}_{r P_{n-2} \cap P_{n-2}}^{P_{n-2}}\left({ }^{r} \nu_{0}\right)$, where $r=s_{m-1}$ and $\nu_{0}$ is viewed as a character of ${ }^{r} P_{n-2} \cap P_{n-2}$ via inflation.

(d) Let $\nu$ be an irreducible character of $P_{n-2}$ of Type 3, say $\nu={ }^{3} \psi_{\vartheta_{0}^{\prime}}^{i, \varepsilon}$ for some $i \in\{1,2\}$, some $\varepsilon \in\{+,-\}$, and some $\vartheta_{0}^{\prime} \in \operatorname{Irr}\left(L_{n-2}^{\prime}\right)$. Let $\vartheta_{0}:=1_{A_{n-2}}^{-} \otimes \vartheta_{0}^{\prime} \in \operatorname{Irr}\left(L_{n-2}\right)$, and put

$$
\vartheta:=R_{L_{n-2}}^{L^{\prime}}\left(\vartheta_{0}\right)
$$

Then

$$
\operatorname{Ind}_{R Q_{K}}^{P}\left({ }^{s} \nu\right)={ }^{3} \psi_{\vartheta}^{i, \varepsilon}
$$

Proof. For the sake of legibility, we suppress the symbol for inflation in the following proof.

(a) Since $t$ centralizes $L^{\prime}$, we have $\operatorname{Res}_{L}^{t} P\left({ }^{t} \sigma\right)=\sigma$.

(b) We first consider the special case that $A_{n-2}$ is in the kernel of $\nu$. Then ${ }^{s} \nu=\nu$ since $s$ centralizes $L_{n-2}^{\prime}$. Let $\tilde{\nu}$ denote the irreducible character of $\tilde{P}_{n-2}$ corresponding to $\nu$. (See the notation of $[1,2.3 .2]$, 
an account of which is given in Section 2.) We have

$$
\begin{aligned}
\left(\operatorname{Ind}_{U \tilde{P}_{n-2}}^{U Q_{K}}(\hat{\lambda} \cdot \tilde{\nu}), \operatorname{Ind}_{R Q_{K}}^{U Q_{K}}(\nu)\right) & =\left(\hat{\lambda} \cdot \tilde{\nu}, \operatorname{Res}_{U \tilde{P}_{n-2}}^{U Q_{K}}\left(\operatorname{Ind}_{R Q_{K}}^{U Q_{K}}(\nu)\right)\right) \\
& =\left(\hat{\lambda} \cdot \tilde{\nu}, \operatorname{Ind}_{R \tilde{P}_{n-2}}^{U \tilde{P}_{n-2}}\left(\operatorname{Res}_{R \tilde{P}_{n-2}}^{R Q_{K}}(\nu)\right)\right) \\
& =\left(\operatorname{Res}_{R \tilde{P}_{n-2}}^{U \tilde{P}_{n-2}}(\hat{\lambda}) \cdot \tilde{\nu}, \tilde{\nu}\right) \\
& =(\tilde{\nu}, \tilde{\nu})=1,
\end{aligned}
$$

since $R \leq U$ is in the kernel of $\hat{\lambda}$ and $\operatorname{Res}_{\tilde{P}_{n-2}}^{U \tilde{P}_{n-2}}(\hat{\lambda})=1_{\tilde{P}_{n-2}}$.

Thus ${ }^{2} \psi_{\nu}=\operatorname{Ind}_{U \tilde{P}_{n-2}}^{P}(\hat{\lambda} \cdot \tilde{\nu})$ is a constituent of $\operatorname{Ind}_{R Q_{K}}^{P}(\nu)$. Also, $\nu$ is a constituent of $\operatorname{Ind}_{R Q_{K}}^{U Q_{K}}(\nu)$, hence ${ }^{1} \psi_{\Sigma}$ is a subcharacter of $\operatorname{Ind}_{R Q_{K}}^{P}(\nu)$. The result follows by comparing degrees.

Now we deal with the general case. Write $\nu=1_{A} \otimes \xi \otimes \nu^{\prime}$ with irreducible characters $\xi$ of $A_{n-2}$ and $\nu^{\prime}$ of $L_{n-2}^{\prime}$. Then ${ }^{s} \nu={ }^{s} \xi \bigotimes 1_{A_{n-2}} \otimes \nu^{\prime}$. Viewing ${ }^{s} \xi$ as a linear character of $P$ via inflation, we obtain

$$
\operatorname{Ind}_{R Q_{K}}^{P}\left({ }^{s} \nu\right)={ }^{s} \xi \cdot\left({ }^{1} \psi_{\Sigma^{\prime}}+{ }^{2} \psi_{\nu^{\prime}}\right),
$$

with $\Sigma^{\prime}=R_{L_{K}}^{L}\left(\nu^{\prime}\right)$. The result follows from the observations ${ }^{s} \xi \cdot{ }^{1} \psi_{\Sigma^{\prime}}=$ ${ }^{1} \psi_{\Sigma}$ and ${ }^{s} \xi \cdot{ }^{2} \psi_{\nu^{\prime}}={ }^{2} \psi_{\nu}$.

(c) We first show that $Z(U)$ is in the kernel of $\operatorname{Ind}_{R Q_{K}}^{P}\left({ }^{s} \nu\right)$. Indeed, $Z(U)={ }^{s}\left(Z\left(U_{n-2}\right)\right)$, and so $Z(U)$ is in the kernel of ${ }^{s} \nu$. Hence $Z(U)$ is in the kernel of $\operatorname{Ind}_{R Q_{K}}^{P}\left(s_{\nu}\right)$.

Next we show that $\operatorname{Ind}_{R Q_{K}}^{P}\left({ }^{s} \nu\right)$ does not have any constituents of Type 1. We have

$$
\left(\operatorname{Res}_{U}^{P}\left(\operatorname{Ind}_{R Q_{K}}^{P}\left({ }^{s} \nu\right)\right), 1_{U}\right)=\left({ }^{s} \nu, \operatorname{Res}_{R Q_{K}}^{P}\left(\operatorname{Ind}_{U}^{P}\left(1_{U}\right)\right)\right)
$$

and every constituent of $\operatorname{Res}_{R Q_{K}}^{P}\left(\operatorname{Ind}_{U}^{P}\left(1_{U}\right)\right)$ has $R$ in its kernel. On the other hand, ${ }^{s} L \cap U \leq R$, and so ${ }^{s} U \cap L \leq{ }^{s} R$. This implies that $R$ is not in the kernel of ${ }^{s} \nu$, since ${ }^{s} U \cap L$, the unipotent radical of $P_{n-1}$, is not in the kernel of $\nu$ by assumption. This implies that the scalar product in Equation (4) is zero, and hence $\operatorname{Ind}_{R Q_{K}}^{P}\left({ }^{s} \nu\right)$ does not have any constituent with $U$ in its kernel.

Thus $\operatorname{Ind}_{R Q_{K}}^{P}\left({ }^{\nu} \nu\right)$ only contains constituents of Type 2 .

Let $\lambda_{n-2}$ be the irreducible character of $U_{n-2}$ analogous to $\lambda$, and let $\hat{\lambda}_{n-2}$ be the extension of $\lambda_{n-2}$ to $U_{n-2} \tilde{P}_{n-4}$, with $\operatorname{Res}_{\tilde{P}_{n-4}}^{U_{n-2} \tilde{P}_{n-4}}\left(\hat{\lambda}_{n-2}\right)=$ $1_{\tilde{P}_{n-4}}$. Then, by definition,

$$
\nu=\operatorname{Ind}_{U_{n-2} \tilde{P}_{n-4}}^{P_{n-2}}\left(\hat{\lambda}_{n-2} \cdot \operatorname{Infl}_{\tilde{P}_{n-4}}^{U_{n-2} \tilde{P}_{n-4}}\left(\tilde{\nu}_{0}\right)\right) .
$$


We put $Q_{K}^{\prime}:=A \times U_{n-2} \tilde{P}_{n-4} \leq Q_{K}$, and inflate the characters in Equation (5) over the normal subgroup $R A$ of $R Q_{K}^{\prime}$ and $R Q_{K}$. Suppressing the symbols for inflation, this yields

$$
\nu=\operatorname{Ind}_{R Q_{K}^{\prime}}^{R Q_{K}}\left(\hat{\lambda}_{n-2} \cdot \tilde{\nu}_{0}\right) .
$$

Since $r \in P$, we obtain

$$
\operatorname{Ind}_{R Q_{K}}^{P}\left({ }^{s} \nu\right)={ }^{r} \operatorname{Ind}_{R Q_{K}}^{P}\left({ }^{s} \nu\right)=\operatorname{Ind}_{r s\left(R Q_{K}^{\prime}\right)}^{P}\left({ }^{r} \hat{\lambda}_{n-2} \cdot{ }^{r s} \tilde{\nu}_{0}\right) .
$$

Put $\widetilde{\Sigma}:=\operatorname{Res}_{\tilde{P}_{n-2}}^{A \times P_{n-2}}\left(1_{A} \otimes \Sigma\right)$. Then ${ }^{2} \psi_{\Sigma}=\operatorname{Ind}_{U \tilde{P}_{n-2}}^{P}\left(\hat{\lambda} \cdot \operatorname{Infl}_{\tilde{P}_{n-2}}^{U \tilde{P}_{n-2}}(\widetilde{\Sigma})\right.$. We claim that $\hat{\lambda} \cdot \operatorname{Infl}_{\tilde{P}_{n-2}}^{U \tilde{P}_{n-2}}(\widetilde{\Sigma})$ is a subcharacter of $\operatorname{Res}_{U \tilde{P}_{n-2}}^{P}\left(\operatorname{Ind}_{R Q_{K}}^{P}\left({ }^{s} \nu\right)\right)$. By Frobenius reciprocity, this would imply that ${ }^{2} \psi_{\Sigma}$ is a subcharacter of $\operatorname{Ind}_{R Q_{K}}^{P}\left({ }^{s} \nu\right)$, since every irreducible constituent of $\hat{\lambda} \cdot \operatorname{Infl}_{\tilde{P}_{n-2}}^{U \tilde{P}_{n-2}}(\widetilde{\Sigma})$ induces to an irreducible Type 2 character of $P$. Since $\operatorname{Ind}_{R Q_{K}}^{P}\left({ }^{s} \nu\right)$ and ${ }^{2} \psi_{\Sigma}$ have the same degree, this would imply the desired result.

So it suffices to prove the claim. By Mackey's theorem and Equation (7), it suffices to show that $\hat{\lambda} \cdot \operatorname{Infl}_{\tilde{P}_{n-2}}^{U \tilde{P}_{n-2}}(\widetilde{\Sigma})$ is a subcharacter of

$$
\operatorname{Ind}_{U \tilde{P}_{n-2} \cap^{r s}\left(R Q_{K}^{\prime}\right)}^{U \tilde{P}_{n-2}}\left(\operatorname{Res}_{U \tilde{P}_{n-2} \cap^{r s}\left(R Q_{K}^{\prime}\right)}\left(R Q_{K}^{\prime}\right)\left({ }^{r s} \hat{\lambda}_{n-2} \cdot{ }^{r s} \tilde{\nu}_{0}\right)\right)
$$

By Lemma 3.2 we have

$$
U \tilde{P}_{n-2} \cap{ }^{r s}\left(R Q_{K}^{\prime}\right)=\left({ }^{r} R\right) Y
$$

with $Y \leq \tilde{P}_{n-2}$ such that $A \times Y=A \times\left({ }^{r} P_{n-2} \cap P_{n-2}\right)$.

Now ${ }^{r s} U_{n-2} \leq U$, and ${ }^{r} \hat{\lambda}_{n-2}$ and $\lambda$ agree on this subgroup of $U$. Clearly ${ }^{r} R$ is in the kernel of ${ }^{r} \tilde{\nu}_{0}$, and so ${ }^{r s} \hat{\lambda}_{n-2} \cdot{ }^{r} \tilde{\nu}_{0}$ extends to the character $\hat{\lambda} \cdot r \tilde{\nu}_{0}$ of $U Y$. In particular, $\hat{\lambda} \cdot{ }^{r s^{2}} \tilde{\nu}_{0}$ is a constituent of $\operatorname{Ind}_{\left({ }^{r} R\right) Y}^{U Y}\left({ }^{r s} \hat{\lambda}_{n-2} \cdot{ }^{r} \tilde{\nu}_{0}\right)$. Since $\lambda$ extends to $\hat{\lambda} \in \operatorname{Irr}\left(U \tilde{P}_{n-2}\right)$, it follows that $\hat{\lambda} \cdot \operatorname{Ind}_{Y}^{\tilde{P}_{n-2}}\left({ }^{r} \tilde{\nu}_{0}\right)$ is a subcharacter of $\operatorname{Ind}_{\left({ }^{r} R\right) Y}^{U \tilde{P}_{n-2}}\left({ }^{r s} \hat{\lambda}_{n-2} \cdot{ }^{r} \tilde{\nu}_{0}\right)$. Since $\operatorname{Ind}_{Y}^{\tilde{P}_{n-2}}\left({ }^{r} \tilde{\nu}_{0}\right)=\widetilde{\Sigma}$, our claim follows.

(d) We have $Z(U) \leq{ }^{s} L \cap U \leq R$, and so a similar argument as in (c) with $U$ replaced by $Z(U)$ shows that $\operatorname{Ind}_{R Q_{K}}^{P}\left({ }^{s} \nu\right)$ only has constituents of Type 3.

The proof now proceeds in several steps.

Step 1: We identify the subgroup $\left({ }^{s} U \cap L\right) L_{n-2}^{\prime}=U_{n-2} L_{n-2}^{\prime}$ of $R P_{n-2}$ with $P_{n-2}^{\prime}$. Clearly, $s$ centralizes $L_{n-2}^{\prime}$ and ${ }^{s}\left(\left({ }^{s} U \cap L\right) L_{n-2}^{\prime}\right)=$ $\left({ }^{s} L \cap U\right) L_{n-2}^{\prime}$. Let $\beta$ be the irreducible character of degree $q^{m-2}$ of $\left({ }^{s} U \cap L\right) L_{n-2}^{\prime}=P_{n-2}^{\prime}$ such that ${ }^{s} \beta$ lies over $\zeta_{i}$, i.e., $\beta\left(\mathbf{u}_{n-2}(0, z)\right)=\zeta_{i}(z)$ for $z \in \mathbb{F}_{q}^{*}$. Now $A_{n-2}$ centralizes $\left({ }^{s} L \cap U\right) L_{n-2}^{\prime}$ and

$$
\left({ }^{s} L \cap U\right) L_{n-2}=A_{n-2} \times\left({ }^{s} L \cap U\right) L_{n-2}^{\prime} .
$$


Moreover, $\left({ }^{s} U \cap U\right)\left({ }^{s} U \cap L\right)$ is a normal subgroup of $R P_{n-2}$ with quotient group isomorphic to $\left({ }^{s} L \cap U\right) L_{n-2}$. Viewing $1_{A_{n-2}}^{-} \nabla^{s} \beta$ as a character of $R P_{n-2}$ via inflation, we obtain

$$
\operatorname{Res}_{U P_{n-2}}^{U L^{\prime}}\left(\hat{\rho}_{i}\right)=\operatorname{Ind}_{R P_{n-2}}^{U P_{n-2}}\left(1_{A_{n-2}}^{-} \otimes^{s} \beta\right) .
$$

Proof of Step 1: This is exactly Statement (b) of [5, Theorem 2.4]. Notice that our $P_{n-2}$ corresponds to $P\left(E_{+}, j\right)$, our $U$ to $H(E, j)$ and our $R$ to $H\left(E_{+}^{\perp}, j\right)$ of [5], where $E_{+}$is the subspace of $\mathbb{F}_{q}^{n-2}$ spanned by the first standard basis vector.

Step 2: Let $\xi:=\beta \cdot \vartheta_{0}^{\prime}$ where we view $\vartheta_{0}^{\prime}$ as a character of $P_{n-2}^{\prime}$ via inflation over $U_{n-2}$. Then $\xi$ is a constituent of $\operatorname{Res}_{P_{n-2}^{\prime}}^{P_{n-2}}(\nu)$. Again viewing $1_{A_{n-2}} \otimes s^{s}$ as a character of $R P_{n-2}$ via inflation, we obtain

$$
\operatorname{Ind}_{R P_{n-2}}^{U P_{n-2}}\left(1_{A_{n-2}} \otimes^{s} \xi\right)=\operatorname{Res}_{U P_{n-2}}^{U L^{\prime}}\left(\hat{\rho}_{i}\right) \cdot \vartheta_{0}
$$

where $\vartheta_{0}$ is viewed as a character of $U P_{n-2}$ via inflation over $U U_{n-2}$.

Proof of Step 2: The first assertion is clear by the construction of the Type 3 characters of $P_{n-2}$. Using Step 1 we obtain

$$
\begin{aligned}
\operatorname{Res}_{U P_{n-2}}^{U L^{\prime}}\left(\hat{\rho}_{i}\right) \cdot \vartheta_{0} & =\operatorname{Ind}_{R P_{n-2}}^{U P_{n-2}}\left(1_{A_{n-2}}^{-} \otimes^{s} \beta\right) \cdot \vartheta_{0} \\
& =\operatorname{Ind}_{R P_{n-2}}^{U P_{n-2}}\left(\left(1_{A_{n-2}} \otimes^{s} \beta\right) \cdot \vartheta_{0}\right) .
\end{aligned}
$$

It remains to observe that $1_{A_{n-2}} \otimes s \xi=\left(1_{A_{n-2}}^{-} \otimes^{s} \beta\right) \cdot \vartheta_{0}$ as characters of $R P_{n-2}$.

Step 3: $\operatorname{Ind}_{R P_{n-2}}^{U L^{\prime}}\left(1_{A_{n-2}} \otimes^{s} \xi\right)=\hat{\rho}_{i} \cdot \operatorname{Infl}_{L^{\prime}}^{U L^{\prime}}(\vartheta)$, where $\vartheta$ is viewed as a character of $U L^{\prime}$ via inflation over $U$.

Proof of Step 3: From Step 2 we obtain

$$
\begin{aligned}
\operatorname{Ind}_{R P_{n-2}}^{U L^{\prime}}\left(1_{A_{n-2}} \otimes^{s} \xi\right) & =\operatorname{Ind}_{U P_{n-2}}^{U L^{\prime}}\left(\operatorname{Ind}_{R P_{n-2}}^{U P_{n-2}}\left(1_{A_{n-2}} \otimes^{s} \xi\right)\right) \\
& =\operatorname{Ind}_{U P_{n-2}}^{U L^{\prime}}\left(\operatorname{Res}_{U P_{n-2}}^{U L^{\prime}}\left(\hat{\rho}_{i}\right) \cdot \vartheta_{0}\right) \\
& =\hat{\rho}_{i} \cdot \operatorname{Ind}_{U P_{n-2}}^{U L^{\prime}}\left(\vartheta_{0}\right) \\
& =\hat{\rho}_{i} \cdot \operatorname{Ind}_{P_{n-2}}^{L^{\prime}}\left(\vartheta_{0}\right) \\
& =\hat{\rho}_{i} \cdot R_{L_{n-2}}^{L^{\prime}}\left(\vartheta_{0}\right) \\
& =\hat{\rho}_{i} \cdot \vartheta
\end{aligned}
$$

Step 4: $\operatorname{Ind}_{R Q_{K}}^{P}\left({ }^{s} \nu\right)={ }^{3} \psi_{\vartheta}^{i, \varepsilon}$.

Proof of Step 4: We have

$$
\operatorname{Res}_{U L^{\prime} \times Z}^{P}\left(\operatorname{Ind}_{R Q_{K}}^{P}\left({ }^{s} \nu\right)\right)=\operatorname{Ind}_{R P_{n-2} \times Z}^{U L^{\prime} \times Z}\left(\operatorname{Res}_{R P_{n-2} \times Z}^{R Q_{K}}\left({ }^{s} \nu\right)\right) .
$$


Now $\left(1_{A_{n-2}} \otimes s^{s} \xi\right) \otimes 1_{Z}^{\varepsilon}$ is an irreducible constituent of $\operatorname{Res}_{R P_{n-2} \times Z}^{R Q_{K}}\left({ }^{s} \nu\right)$, and hence $\left(\hat{\rho}_{i} \cdot \operatorname{Infl}_{L^{\prime}}^{U L^{\prime}}(\vartheta)\right) \otimes 1_{Z}^{\varepsilon}$ is a subcharacter of $\operatorname{Res}_{U L^{\prime} \times Z}^{P}\left(\operatorname{Ind}_{R Q_{K}}^{P}\left(s_{\nu}\right)\right)$ by Step 3. Clifford theory and the construction of ${ }^{3} \psi_{\vartheta}^{i, \varepsilon}$ now proves the claim in Step 4.

Remark 3.4. Note that $\operatorname{Ind}_{L}^{P}(\sigma)$ has been computed in [1, Proposition 3.2]. There is a slight inaccuracy in the formulation of this result in [1]. With the notation introduced above, the correct statement is as follows:

$$
\begin{aligned}
\operatorname{Ind}_{L}^{P}(\sigma) & ={ }^{1} \psi_{\sigma} \\
& +\sum_{\mu \in \operatorname{Irr}\left(P_{n-2}\right)}\left\langle\operatorname{Res}_{P_{n-2}}^{L^{\prime}}(\sigma), \mu\right\rangle_{P_{n-2}}{ }^{2} \psi_{\mu} \\
& +\sum_{\vartheta \in \operatorname{Irr}\left(L^{\prime}\right)}\left\langle\sigma \cdot \bar{\omega}_{1}, \vartheta\right\rangle_{L^{\prime}}{ }^{3} \psi_{\vartheta}^{1,+}+\left\langle\sigma \cdot \bar{\omega}_{2}, \vartheta\right\rangle_{L^{\prime}}{ }^{3} \psi_{\vartheta}^{2,+} .
\end{aligned}
$$

The difference is in the occurrence of the $\bar{\omega}_{i}$ in the third summand, but this is exactly what we prove in [1].

Corollary 3.5. Suppose that $n \geq 4$. Then

$$
\operatorname{Res}_{P}^{G}\left(R_{L}^{G}\left(1_{L}\right)\right)=2 \cdot{ }^{1} \psi_{1_{L}}+{ }^{1} \psi_{\Sigma}+2 \cdot{ }^{2} \psi_{1_{P_{n-2}}}+{ }^{3} \psi_{\bar{\omega}_{1}}^{1,+}+{ }^{3} \psi_{\bar{\omega}_{2}}^{2,+},
$$

where $\Sigma=R_{L_{K}}^{L}\left(1_{L_{K}}\right)$. (Notice that ${ }^{1} \psi_{\Sigma}$ contains another trivial constituent ${ }^{1} \psi_{1_{L}}=1_{P}$.)

Proof. One constituent ${ }^{1} \psi_{1_{L}}=1_{P}$ arises from the $P$ - $P$-double coset representative 1. The representative $t$ yields the summand $\operatorname{Ind}_{L}^{P}\left(1_{L}\right)=$ ${ }^{1} \psi_{1_{L}}+{ }^{2} \psi_{1_{P_{n-2}}}+{ }^{3} \psi_{\bar{\omega}_{1}}^{1,+}+{ }^{3} \psi_{\bar{\omega}_{2}}^{2,+}$ by Theorem 3.3(a) and Remark 3.4. Finally, $s$ gives the summand ${ }^{1} \psi_{\Sigma}+{ }^{2} \psi_{1_{P_{n-2}}}$ by Theorem 3.3(b).

\section{VAlues of SOME Characters of $P$ ON $U$}

Before we begin to compute the restrictions of the unipotent characters of $G$ to $P$ for $G=\operatorname{Sp}_{4}(q)$ and $\operatorname{Sp}_{6}(q)$, we collect some facts about values of certain characters of $P$ on its unipotent radical $U$. In the following, we use the notation of Section 2 for the irreducible characters of $P$.

The non-trivial elements of $Z(U)$ fall into two $P$-conjugacy classes, $c_{1}:=\left\{\mathbf{u}_{n}(0, z) \mid 0 \neq z \in \mathbb{F}_{q}\right.$ is a square $\}$, and $c_{2}:=\left\{\mathbf{u}_{n}(0, z) \mid 0 \neq z \in\right.$ $\mathbb{F}_{q}$ is a non-square $\}$. Fix elements $\mathbf{z}_{i} \in c_{i}$, and let $C_{i}$ be the conjugacy class of $G$ containing $\mathbf{z}_{i}, i=1,2$. Then $C_{1} \neq C_{2}$. Indeed, since $P$ is 
a maximal subgroup of $G$ and since $P$ normalizes $\left\langle\mathbf{z}_{i}\right\rangle$, it follows that $N_{G}\left(\left\langle\mathbf{z}_{i}\right\rangle\right)=P$ for $i=1,2$. But $c_{1} \neq c_{2}$, so $C_{1} \neq C_{2}$.

There is an automorphism $\alpha:=\alpha_{n}$ of $G$, which fixes $P$ and interchanges $c_{1}$ and $c_{2}$, and thus $C_{1}$ and $C_{2}$. (In particular, $\alpha$ is not an inner automorphism.) To be specific, we may take

$$
\alpha_{n}(\mathbf{x}):=\tilde{J}_{n}(a)\left(\mathbf{x}^{-1}\right)^{t} \tilde{J}_{n}(a)^{-1}, \quad \mathbf{x} \in G,
$$

where $a \in \mathbb{F}_{q}$ is a fixed non-square, and $\tilde{J}_{n}(a)$ is the matrix

$$
\tilde{J}_{n}(a):=\left[\begin{array}{cc}
0 & J_{m} \\
-a^{-1} J_{m} & 0
\end{array}\right] .
$$

Clearly, $\alpha$ permutes the irreducible characters of each type among themselves.

In Section 2 we have chosen two irreducible characters $\zeta_{1}$ and $\zeta_{2}$ representing the two orbits of $P$ on the set of non-trivial irreducible characters of $Z(U)$. We may choose notation such that $\zeta_{2}={ }^{\alpha} \zeta_{1}$. Then the two irreducible characters $\rho_{i}$ of $U$ lying above $\zeta_{i}$ are also conjugate by $\alpha$, as are their extensions $\hat{\rho}_{i}$ to their inertia subgroup $P^{\prime} Z$. It is easy to check that $\alpha$ centralizes $A$ and fixes $L^{\prime}$, where it induces the automorphism $\alpha_{n-2}$, also denoted by $\alpha$. This implies that

$$
\alpha\left({ }^{3} \psi_{\vartheta}^{1, \varepsilon}\right)={ }^{3} \psi_{\alpha \vartheta}^{2, \varepsilon},
$$

for all $\vartheta \in \operatorname{Irr}\left(L^{\prime}\right)$ and all $\varepsilon \in\{+,-\}$. If $\vartheta$ is a character of $L^{\prime}$, we put

$$
{ }^{3} \tilde{\psi}_{\vartheta}^{\varepsilon}:={ }^{3} \psi_{\vartheta}^{1, \varepsilon}+{ }^{3} \psi_{\alpha \vartheta}^{2, \varepsilon} \text {. }
$$

It follows from the transitivity of $P$ on $U / Z(U) \backslash\{Z(U)\}$ and on $Z(U) \backslash\{1\}$ that $U \backslash Z(U)$ is a single conjugacy class of $P$. Let $\mathbf{u} \in$ $U \backslash Z(U)$.

Lemma 4.1. Let $\sigma, \mu$ and $\vartheta$ be characters of $L, P_{n-2}$ and $L^{\prime}$, respectively. Then the corresponding characters of $P$ have the following values on $U$.

\begin{tabular}{c||c|c|c}
\hline Character & 1 & $\mathbf{z}_{i}$ & $\mathbf{u}$ \\
\hline${ }^{1} \psi_{\sigma}$ & $\sigma(1)$ & $\sigma(1)$ & $\sigma(1)$ \\
${ }^{2} \psi_{\mu}$ & $\left(q^{n-2}-1\right) \mu(1)$ & $\left(q^{n-2}-1\right) \mu(1)$ & $-\mu(1)$ \\
${ }^{3} \tilde{\psi}_{\vartheta}^{\varepsilon}$ & $q^{m-1}(q-1) \vartheta(1)$ & $-q^{m-1} \vartheta(1)$ & 0 \\
\hline
\end{tabular}

Proof. The first row and the first column of the character values are clear. Let ${ }^{2} \psi_{\mu}$ be a Type 2 irreducible character of $P$ with $\mu \in$ $\operatorname{Irr}\left(P_{n-2}\right)$; then $Z(U)$ lies in the kernel of ${ }^{2} \psi_{\mu}$ which gives the entry in the second row at $\mathbf{z}_{i}$. Now ${ }^{2} \psi_{\mu}$ is a character of $\bar{P}:=P / Z(U)$. If $\bar{U}:=U / Z(U)$, then $\bar{U}$ is a normal subgroup of $\bar{P}$ of order $q^{n-2}$. Since 
$\bar{P}$ acts transitively on the non-trivial irreducible characters of $\bar{U}$, it follows that

$$
\operatorname{Res}_{\bar{U}}^{\bar{P}}\left({ }^{2} \psi_{\mu}\right)=e \sum_{\lambda \in \operatorname{Irr}(\bar{U}) \backslash\left\{1_{\bar{U}}\right\}} \lambda
$$

for some integer $e \geq 1$. Thus ${ }^{2} \psi_{\mu}(\mathbf{u})={ }^{2} \psi_{\mu}(\mathbf{u} Z(U))=-e$. But

$$
\left(q^{n-2}-1\right) \mu(1)={ }^{2} \psi_{\mu}(1)=e\left(q^{n-2}-1\right),
$$

so $e=\mu(1)$ and ${ }^{2} \psi_{\mu}(\mathbf{u})=-\mu(1)$.

To prove the third row, let ${ }^{3} \psi_{\vartheta}^{i, \varepsilon}$ be a Type 3 irreducible character of $P$, where $\vartheta \in \operatorname{Irr}\left(L^{\prime}\right), i \in\{1,2\}$ and $\varepsilon \in\{+,-\}$. By construction, $\rho_{i}$ is a constituent of $\operatorname{Res}_{U}^{P}\left({ }^{3} \psi_{\vartheta}^{i, \varepsilon}\right)$. Since $\operatorname{Res}_{Z(U)}^{U}\left(\rho_{i}\right)=q^{m-1} \zeta_{i}$, the orthogonality relations imply that $\rho_{i}(\mathbf{u})=0$ and thus

$$
{ }^{3} \psi_{\vartheta}^{i, \varepsilon}(\mathbf{u})=0 .
$$

Finally, let $\bar{A}$ denote a set of representatives for the quotient of $A$ modulo its subgroup of order 2 . Then the restriction of $\psi_{\vartheta}^{1, \varepsilon}$ to $U$ equals

$$
\vartheta(1) \sum_{a \in \bar{A}}{ }^{a} \rho_{1},
$$

by the construction of ${ }^{3} \psi_{\vartheta}^{1, \varepsilon}$ (see $[1,2.3 .3]$ or Section 2). Similarly, the restriction to $U$ of $3 \psi_{\alpha \vartheta}^{2, \varepsilon}$ equals

$$
\vartheta(1) \sum_{a \in \bar{A}}{ }^{a} \rho_{2} .
$$

Hence the restriction of ${ }^{3} \psi_{\vartheta}^{1, \varepsilon}+{ }^{3} \psi_{\alpha_{\vartheta}}^{2, \varepsilon}$ to $Z(U)$ equals

$$
\vartheta(1) q^{m-1} \sum_{\zeta \in \operatorname{Irr}(Z(U)) \backslash\left\{1_{Z(U)}\right\}} \zeta,
$$

which has the claimed value on the $\mathbf{z}_{i}$.

This result can be used to obtain some information about the components of the various types in a restricted $\alpha$-invariant character.

Lemma 4.2. Let $\chi$ be a character of $G$. Write

$$
\operatorname{Res}_{P}^{G}(\chi)={ }^{1} \chi+{ }^{2} \chi+{ }^{3} \chi
$$

where ${ }^{i} \chi$ denotes the sum of the constituents of Type $i$ in $\operatorname{Res}_{P}^{G}(\chi)$, $i=1,2,3$. We call ${ }^{i} \chi$ the Type $i$ component of $\operatorname{Res}_{P}^{G}(\chi)$.

If $\chi$ is $\alpha$-invariant, then

$$
{ }^{1} \chi(1)+{ }^{2} \chi(1)=\frac{1}{q}(\chi(1)+(q-1) \chi(\mathbf{z}))
$$


and

$$
{ }^{3} \chi(1)=\frac{q-1}{q}(\chi(1)-\chi(\mathbf{z})),
$$

where $\mathbf{z}$ is a non-trivial element in the center of $U$.

Proof. We have ${ }^{i} \chi(1)={ }^{i} \chi(\mathbf{z})$ for $i=1,2$ by Lemma 4.1. Also, since $\chi$ is $\alpha$-invariant, the constituents of Type 3 of $\operatorname{Res}_{P}^{G}(\chi)$ occur in $\alpha$-conjugate pairs. From (9) and Lemma 4.1 we find

$$
{ }^{3} \chi(1)=-{ }^{3} \chi(\mathbf{z})(q-1) .
$$

The claim follows from the two equations

$$
\chi(1)={ }^{1} \chi(1)+{ }^{2} \chi(1)+{ }^{3} \chi(1)
$$

and

$$
\chi(\mathbf{z})={ }^{1} \chi(1)+{ }^{2} \chi(1)-\frac{1}{q-1}^{3} \chi(1) .
$$

We record one further useful result.

Lemma 4.3. Let $\chi \in \operatorname{Irr}(G)$ such that $Z(G)$ is in the kernel of $\chi$. (This is the case if $\chi$ is unipotent.) If $\vartheta \in \operatorname{Irr}\left(L^{\prime}\right)$ such that ${ }^{3} \psi_{\vartheta}^{i, \varepsilon}$ occurs in $\operatorname{Res}_{P}^{G}(\chi)$ for some $i \in\{1,2\}$ and $\varepsilon \in\{+,-\}$, then $\varepsilon=+$.

Proof. Every irreducible constituent of $\operatorname{Res}_{P}^{G}(\chi)$ has $Z(G)$ in its kernel. By construction, this is not the case for the characters ${ }^{3} \psi_{\vartheta}^{i,-}$, $i=1,2$.

\section{The Restrictions of the unipotent Characters of $\operatorname{Sp}_{4}(q)$}

Let $\chi$ be a unipotent character of $G=\operatorname{Sp}_{n}(q)$. (We adopt the convention that the adjective "unipotent" implies irreducible.) Then $\chi$ is labeled by a symbol $\Lambda$. We will identify a symbol $\Lambda$ with a triple $[\mu, \lambda, d]$, where $d$ is an odd, positive integer and $\mu$ and $\lambda$ are partitions such that $|\mu|+|\lambda|+\left(d^{2}-1\right) / 4=m$ (see $[2$, Sections 11.4, 13.2 and 13.8]). Here, $|\mu|$ denotes the sum of the parts of $\mu$.

Suppose that $n=4$, so that $G=\operatorname{Sp}_{4}(q)$. Then $G$ has 6 unipotent characters. In this section we determine their restrictions to $P=P_{4}$. In the course of the proof we are going to refer to the character table of $G$ determined by Srinivasan in [10] for some character values as well as for the notation for certain irreducible characters of $G$. The latter also becomes relevant in the next section where we consider $\operatorname{Sp}_{6}(q)$ and its parabolic subgroup, some of whose characters are labelled by 
irreducible characters of $\operatorname{Sp}_{4}(q)$. We thus have to match the Weil characters $\omega_{1}$ and $\omega_{2}$ with the Weil characters of Srinivasan's character table.

First note that the conjugacy class $C_{1}$ introduced in Section 4 is called $A_{21}$ in [10]. The Weil characters of [10] are denoted by $\theta_{7}+\theta_{3}$ and $\theta_{8}+\theta_{4}$ (the sum $\theta_{8}+\theta_{3}$ is not a Weil character, since its value on the class $A_{21}$ equals zero). We now choose our character $\zeta_{1} \in \operatorname{Irr}\left(\mathbb{F}_{q}\right)$ in such a way that $\bar{\omega}_{1}=\theta_{7}+\theta_{3}$.

The following lemma serves two purposes. Firstly, we introduce a notation for the irreducible Weil characters of $L^{\prime}=\operatorname{Sp}_{2}(q)$, and secondly we collect some facts about Harish-Chandra induced characters of $\operatorname{Sp}_{4}(q)$, needed in the course of the proof of our main result in the following section. We write ${ }^{*} R_{L}^{G}$ for Harish-Chandra restriction from $G$ to $L$. Thus ${ }^{*} R_{L}^{G}={ }^{1} \chi$ in the notation of Lemma 4.2.

Lemma 5.1. Let $G=\operatorname{Sp}_{4}(q)$. Then $L=A \times L^{\prime}$ with $L^{\prime}=\operatorname{Sp}_{2}(q) \cong$ $\mathrm{SL}_{2}(q)$. Let $\nu_{3}, \nu_{7} \in \operatorname{Irr}\left(L^{\prime}\right)$ be defined by

$$
{ }^{*} R_{L}^{G}\left(\theta_{i}\right)=1_{A}^{-} \otimes \nu_{i}, \quad i=3,4,7,8 .
$$

(By [12, Proposition $2.2(\mathrm{v})],{ }^{*} R_{L}^{G}\left(\theta_{i}\right)$ is of this form, and $\nu_{3}, \nu_{7}$ are irreducible Weil characters of $L^{\prime}$ of degrees $(q+1) / 2$ and $(q-1) / 2$, respectively.)

(a) Let $\vartheta=1_{A}^{-} \otimes \nu_{7}$. Then

$$
R_{L}^{G}(\vartheta)=\theta_{5}+\theta_{7}
$$

(b) Let $\vartheta=1_{A}^{-} \otimes \nu_{3}$. Then

$$
R_{L}^{G}(\vartheta)=\Phi_{9}+\theta_{2}+\theta_{3} .
$$

By applying the outer automorphism $\alpha$ of $G$ we obtain $R_{L}^{G}(\vartheta)$ for $\vartheta=$ $1_{A}^{-} \otimes \nu_{8}$ and $\vartheta=1_{A}^{-} \otimes \nu_{4}$.

Proof. (a) Note that $\vartheta$ is a cuspidal character of $L$. The inertia group $W_{G}(L, \vartheta)$ is of order 2 . Thus $R_{L}^{G}(\vartheta)=\chi_{1}+\chi_{2}$ with two distinct irreducible characters $\chi_{1}$ and $\chi_{2}$. By construction, $\theta_{7}$ occurs in $R_{L}^{G}(\vartheta)$. Putting $\chi_{2}:=\theta_{7}$, we have $\chi_{1}(1)=q^{2}\left(q^{2}-1\right) / 2$. Now $\theta_{5}$ and $\theta_{6}$ are the only irreducible characters of $G$ of this degree.

Since $\theta_{7}+\theta_{3}$ is a Weil character of $G$, it follows from [5, Corollary 2,5] that

$$
\operatorname{Res}_{L^{\prime}}^{G}\left(\theta_{7}+\theta_{3}\right)=q\left(\nu_{7}+\nu_{3}\right)
$$

This allows us to compute the values of $\nu_{7}$ and $\nu_{3}$ on the element $\mathbf{z}_{1}^{\prime}={ }^{s} \mathbf{z}_{1} \in L^{\prime}$. The intersection of the $G$-conjugacy class of $\mathbf{z}_{1}$ with $P$ splits into two $P$-conjugacy classes with representatives $\mathbf{z}_{1}$ and $\mathbf{z}_{1}^{\prime}$. We can thus compute the value of $R_{L}^{G}(\vartheta)$ on $\mathbf{z}_{1}$, giving our result. 
(b) This time, $\vartheta$ lies in the Harish-Chandra series of the character $1_{A_{4}}^{-} \otimes 1_{A_{2}}^{-}$of the maximal split torus $A_{4} \times A_{2}$ of $G$. Moreoever, $\vartheta$ corresponds to one of the two irreducible characters of the subgroup $\left\langle s_{1}\right\rangle$ of $W$ (in the sense of [3, Theorem (70.24)]). Inducing these characters to $W$, we obtain exactly three constituents in each case.

The characters of $G$ in the Harish-Chandra series of $G$ corresponding to $\left(L, 1_{A_{4}}^{-} \otimes 1_{A_{2}}^{-}\right)$are $\theta_{i}, i=1, \ldots, 4$, and $\Phi_{9}$, where $\Phi_{9}$ corresponds to the character of degree 2 of $W$ (see [11, Appendix]). Again, by construction, $\theta_{3}$ occurs in $R_{L}^{G}(\vartheta)$. For reasons of degrees, $\Phi_{9}$ also occurs. The remaining constituent is $\theta_{1}$ or $\theta_{2}$. With the same argument as in (a) we conclude that $\theta_{2}$ occurs. This gives the result.

Remark 5.2. For the convenience of the reader we display the values of the irreducible Weil characters of $\operatorname{Sp}_{4}(q)$ and $\operatorname{Sp}_{2}(q)$ on the classes $C_{0}$ containing the identity element, and on the classes $C_{1}$ and $C_{2}$ introduced at the beginning of Section 4. In the following table, we put $\delta:=(-1)^{(q-1) / 2}$.

\begin{tabular}{c|cccc}
\hline & $\theta_{7}$ & $\theta_{3}$ & $\nu_{7}$ & $\nu_{3}$ \\
\hline$C_{0}$ & $\frac{1}{2}\left(q^{2}-1\right)$ & $\frac{1}{2}\left(q^{2}+1\right)$ & $\frac{1}{2}(q-1)$ & $\frac{1}{2}(q+1)$ \\
$C_{1}$ & $\frac{1}{2}(-1-q \delta \sqrt{\delta q})$ & $\frac{1}{2}(1-q \delta \sqrt{\delta q})$ & $\frac{1}{2}(-1-\delta \sqrt{\delta q})$ & $\frac{1}{2}(1-\delta \sqrt{\delta q})$ \\
$C_{2}$ & $\frac{1}{2}(-1+q \delta \sqrt{\delta q})$ & $\frac{1}{2}(1+q \delta \sqrt{\delta q})$ & $\frac{1}{2}(-1+\delta \sqrt{\delta q})$ & $\frac{1}{2}(1+\delta \sqrt{\delta q})$ \\
\hline
\end{tabular}

The main result of this section is as follows.

Theorem 5.3. Let $\chi_{\Lambda}$ be a unipotent character of $G=\operatorname{Sp}_{4}(q)$. Then $\operatorname{Res}_{P}^{G}\left(\chi_{\Lambda}\right)$ is as given in Table 1. We have

$$
\operatorname{Res}_{P_{2}}^{L^{\prime}}\left(\mathrm{St}_{L^{\prime}}\right)=1_{P_{2}}+{ }^{3} \psi_{1}^{1,+}+{ }^{3} \psi_{1}^{2,+} .
$$

(Notice that here 1 denotes the trivial character of the trivial group $L_{2}^{\prime}$ and that ${ }^{3} \psi_{1}^{1,+}$ and ${ }^{3} \psi_{1}^{2,+}$ are the two irreducible characters of $P_{2}$ of degree $(q-1) / 2$ with $Z(G)$ in their kernels.) Also

$$
\left(\nu_{7}+\nu_{3}\right) \cdot \mathrm{St}_{L^{\prime}}=\sum_{\substack{\vartheta \in \operatorname{Irr}\left(\mathrm{SL}_{2}(q)\right) \\ \vartheta(1) \neq 1,(q-1) / 2}} \vartheta .
$$

In particular, $\operatorname{Res}_{P}^{G}\left(\chi_{\Lambda}\right)$ is multiplicity free.

Remarks. (a) Here is a short explanation of how to read Table 1. The first column contains the symbols labelling the unipotent characters of $G$. The decomposition of $\operatorname{Res}_{P}^{G}\left(\chi_{\Lambda}\right)$ is given in the row labelled by $\Lambda$. The second column contains characters $\sigma$ of $L$, described in the 
TABLE 1. Restrictions of unipotent characters of $\operatorname{Sp}_{4}(q)$

\begin{tabular}{|c||c|c|c|}
\hline \multicolumn{1}{|c||}{$\Lambda$} & \multicolumn{3}{|c|}{$\operatorname{Res}_{P}^{G}\left(\chi_{\Lambda}\right)$} \\
\cline { 2 - 4 } & Type 1 & Type 2 & Type 3 \\
\hline \hline$[2,-, 1]$ & {$[1,-, 1]$} & & $\nu_{8}$ \\
{$[-,-, 3]$} & & $1_{P_{2}}$ & $\nu_{7}$ \\
{$\left[1^{2},-, 1\right]$} & {$[1,-, 1]$} & $1_{P_{2}}$ & $\nu_{3}$ \\
{$[1,1,1]$} & {$[1,-, 1]+[-, 1,1]$} & & $\nu_{4}$ \\
{$[-, 2,1]$} & {$[-, 1,1]$} & $\operatorname{Res}_{P_{2}}^{L^{\prime}}\left(\mathrm{St}_{L^{\prime}}\right)$ & $\left(\nu_{7}+\nu_{3}\right) \cdot \mathrm{St}_{L^{\prime}}$ \\
{$\left[-, 1^{2}, 1\right]$} & {$[-, 1,1]$} & & \\
\hline
\end{tabular}

obvious way through symbols, such that ${ }^{1} \psi_{\sigma}$ is the Type 1 component of $\operatorname{Res}_{P}^{G}\left(\chi_{\Lambda}\right)$. Similarly, the third column contains the characters $\mu$ of $P_{2}$ such that ${ }^{2} \psi_{\mu}$ is the Type 2 component of $\operatorname{Res}_{P}^{G}\left(\chi_{\Lambda}\right)$. Finally, the character $\vartheta$ in the last column indicates that ${ }^{3} \tilde{\psi}_{\vartheta}^{+}$is the Type 3 component of $\operatorname{Res}_{P}^{G}\left(\chi_{\Lambda}\right)$. (We will show that the unipotent characters of $G$ are $\alpha$-invariant.)

(b) The decomposition of the characters $\operatorname{Res}_{P_{2}}^{L^{\prime}}\left(\mathrm{St}_{L^{\prime}}\right)$ and $\left(\nu_{7}+\nu_{3}\right) \cdot \mathrm{St}_{L^{\prime}}$ can be found in [1, p. 257] and [1, Section 4], respectively.

Proof. Let $\chi=\chi_{\Lambda}$ be a unipotent character of $G$. If $\chi$ is the trivial character $1_{G}$, then its restriction to $P$ is the trivial character $1_{P}$. If $\chi$ is the Steinberg character $\mathrm{St}_{G}$ of $G$, then its restriction to $P$ is given in $[1$, Example $3.5(\mathrm{c})]$. So we may suppose that $\chi \neq 1_{G}$ and $\chi \neq \mathrm{St}_{G}$.

The constituents of Type 1 in the restrictions are easily computed by the branching rule for the Weyl group of $G$ and a general result of Harish-Chandra theory (see [3, Theorem (70.24)]). In order to simplify the arguments for the constituents of Types 2 and 3, we are going to apply Corollary 3.5. Since

$$
R_{L}^{G}\left(1_{L}\right)=\chi_{[2,-, 1]}+\chi_{\left[1^{2},-, 1\right]}+\chi_{[1,1,1]},
$$

we obtain from Corollary 3.5:

$$
\operatorname{Res}_{P}^{G}\left(\chi_{\left[1^{2},-, 1\right]}+\chi_{[1,1,1]}\right)=2 \cdot{ }^{1} \psi_{1_{L}}+{ }^{1} \psi_{\mathrm{St}_{L}}+2 \cdot{ }^{2} \psi_{1_{P_{n-2}}}+{ }^{3} \psi_{\bar{\omega}_{1}}^{1,+}+{ }^{3} \psi_{\bar{\omega}_{2}}^{2,+} .
$$

Since $\chi_{\left[1^{2},-, 1\right]}$ is the unique constituent of its degree in $R_{L}^{G}\left(1_{L}\right)$, it is $\alpha$-invariant. Hence all unipotent characters of $G$ are $\alpha$-invariant, so we can apply Lemma 4.2. 
Case 1. $\Lambda=\left[1^{2},-, 1\right]$ and $\Lambda=[1,1,1]$.

Let $\chi=\chi_{\Lambda}$ with $\Lambda=\left[1^{2},-, 1\right]$. Then $\chi(1)=q\left(q^{2}+1\right) / 2$. By Harish-Chandra theory we have ${ }^{1} \chi=\chi_{[1,-, 1]}$. In the notation of [10], $\chi=\theta_{12}$. This can be proved as follows: The degrees of $\operatorname{Irr}(G)$ imply that $\chi_{\left[1^{2},-, 1\right]}=\theta_{12}$ or $\theta_{11}$ and $\chi_{[1,1,1]}=\theta_{9}$. By Lemma 4.1, the right hand side of Equation (10) has value $q^{2}+q$ on $\mathbf{z}_{1}$. Since $\theta_{9}\left(\mathbf{z}_{1}\right)=$ $\theta_{9}\left(A_{21}\right)=q(q+1) / 2$, it follows by $(10)$ that $\chi_{\left[1^{2},-, 1\right]}\left(\mathbf{z}_{1}\right)=q(q+1) / 2$ and thus by [10], $\chi_{\left[1^{2},-, 1\right]}=\theta_{12}$. Note this implies that $\chi_{[-, 2,1]}=\theta_{11}$.

Using $\chi\left(\mathbf{z}_{1}\right)=q(q+1) / 2$, it follows from Lemma 4.2 that

$$
{ }^{3} \chi(1)=\frac{1}{2} q(q-1)^{2} \quad \text { and } \quad{ }^{2} \chi(1)=q^{2}-1 .
$$

In view of Equation (10) and the degrees of the characters given in Section 2, this determines the constituents of $\operatorname{Res}_{P}^{G}\left(\chi_{\left[1^{2},-, 1\right]}\right)$, and of $\operatorname{Res}_{P}^{G}\left(\chi_{[1,1,1]}\right)$ completely.

Case 2. $\Lambda=[-, 2,1]$.

Let $\chi=\chi_{\Lambda}$. Then $\chi(1)=q\left(q^{2}+1\right) / 2$. By Harish-Chandra theory we have ${ }^{1} \chi=\chi_{[-, 1,1]}$. In the notation of [10], $\chi=\theta_{11}$, and so $\chi\left(\mathbf{z}_{1}\right)=$ $\theta_{11}\left(A_{21}\right)=-q(q-1) / 2$. It follows from Lemma 4.2 that

$$
{ }^{3} \chi(1)=\frac{1}{2} q\left(q^{2}-1\right) \text { and } \quad{ }^{2} \chi=0 .
$$

In order to determine the Type 3 component, we use Theorem 3.3 with $\sigma=\chi_{[-, 1,1]}$, the Steinberg character of $L^{\prime}$. We have

$$
R_{L}^{G}\left(\chi_{[-, 1,1]}\right)=\chi_{[-, 2,1]}+\chi_{\left[-, 1^{2}, 1\right]}+\chi_{[1,1,1]} .
$$

Since $\operatorname{Res}_{P}^{G}\left(\operatorname{St}_{G}\right)=\operatorname{Ind}_{L}^{P}\left(\operatorname{St}_{L}\right)$, we obtain from (3) and Theorem 3.3(a):

$$
\operatorname{Res}_{P}^{G}\left(\chi_{[-, 2,1]}+\chi_{[1,1,1]}\right)={ }^{1} \psi_{\chi_{[-, 1,1]}}+\operatorname{Ind}_{R Q_{K}}^{P}\left(\operatorname{Res}_{R Q_{K}}^{P}\left(\chi_{[-, 1,1]}\right)\right) .
$$

Now

$$
\operatorname{Res}_{P_{2}}^{L^{\prime}}\left(\mathrm{St}_{L^{\prime}}\right)=1_{P_{2}}+{ }^{3} \psi_{1}^{1,+}+{ }^{3} \psi_{1}^{2,+}
$$

The only Type 3 constituents in (11) arise from the two Type 3 constituents in $\operatorname{Res}_{P_{2}}^{L^{\prime}}\left(\operatorname{St}_{L^{\prime}}\right)$. In this situation, $L_{2}^{\prime}=\{1\}$, and $L_{2}=A_{2}$ is the torus of $L^{\prime}$ of order $q-1$. From Harish-Chandra theory we obtain

$$
R_{L_{2}}^{L^{\prime}}\left(1_{L_{2}}^{-}\right)=\nu_{3}+\nu_{4} \text {. }
$$

Since $\bar{\omega}^{+}=\nu_{3}$ occurs as label for the Type 3 component of $\operatorname{Res}_{P}^{G}\left(\chi_{[1,1,1]}\right)$, the result of Table 1 follows from Theorem 3.3(e). 
Case 3. $\Lambda=[-,-, 3]$.

Let $\chi=\chi_{\Lambda}$ with $\Lambda=[-,-, 3]$. Then $\chi$ is the cuspidal character of $G$, so that ${ }^{1} \chi=0$. In the notation of [10], $\chi=\theta_{10}$, and so $\chi(1)=$ $q(q-1)^{2} / 2$ and $\chi\left(\mathbf{z}_{1}\right)=\theta_{10}\left(A_{21}\right)=-q(q-1) / 2$. By Lemma 4.2,

$$
{ }^{3} \chi(1)=\frac{1}{2} q(q-1)^{2} .
$$

Since $\chi(1)={ }^{3} \chi(1)$, we have ${ }^{2} \chi=0$ and hence $\chi={ }^{3} \chi$. It follows from Lemmas 4.3 and 4.1 that

$$
{ }^{3} \chi={ }^{3} \psi_{\vartheta}^{1,+}+{ }^{3} \psi_{\alpha \vartheta}^{2,+}={ }^{3} \tilde{\psi}_{\vartheta}^{+}
$$

for some character $\vartheta$ of $L^{\prime}$ of degree $(q-1) / 2$.

Suppose $\vartheta$ is not one of the two Weil characters of degree $(q-1) / 2$. Then $\vartheta=(q-1) / 2 \cdot 1_{L^{\prime}}$ since $\nu(1)>(q-1) / 2$ for all $\nu \in \operatorname{Irr}\left(L^{\prime}\right) \backslash$ $\left\{1_{L^{\prime}}, \nu_{7}, \nu_{8}\right\}$ (see $\left.[7]\right)$. Then

$$
\operatorname{Res}_{P}^{G}(\chi)=\frac{1}{2}(q-1)^{3} \tilde{\psi}_{1_{L^{\prime}}}^{+} .
$$

Let $C_{21}(1)$ be the conjugacy class of $G$ described in [10, p. 491]. Then $C_{21}(1) \cap P \neq \emptyset$ and $\chi\left(C_{21}(1)\right)=-1$. This contradicts (12).

It follows that $\vartheta=\nu_{7}$ or $\nu_{8}$. Suppose that $\vartheta=\nu_{7}$, so that by Case 1 ,

$$
{ }^{3} \tilde{\psi}_{\vartheta}^{+}=\operatorname{Res}_{P}^{G}\left(\chi_{\left[1^{2},-, 1\right]}\right)-1_{P}-{ }^{2} \psi_{1_{P_{2}}} .
$$

Put $\mathbf{z}_{1}^{\prime}:={ }^{s} \mathbf{z}_{1}$ with $s$ as in Section 3. Then $\mathbf{z}_{1}^{\prime} \leq Z\left(U_{2}\right) \leq L$, and one computes ${ }^{2} \psi_{1_{P_{2}}}\left(\mathbf{z}_{1}^{\prime}\right)=q-1$. Hence

$$
\left(\operatorname{Res}_{P}^{G}\left(\chi_{\left[1^{2},-, 1\right]}\right)-1_{P}-{ }^{2} \psi_{1_{P_{2}}}\right)\left(\mathbf{z}_{1}^{\prime}\right)=\frac{1}{2} q(q-1) .
$$

On the other hand, $\chi\left(\mathbf{z}_{1}^{\prime}\right)=\theta_{10}\left(A_{21}\right)=-q(q-1) / 2$. This is impossible, so that $\vartheta=\nu_{8}$.

Proposition 5.4. Let $G=\operatorname{Sp}_{4}(q)$ and $t=\frac{1}{2}(q-1)$. We will follow the notation of [10]. In particular, $\theta_{3}$ and $\theta_{7}$ are the irreducible Weil characters of $G$ of degrees $\left(q^{2}+1\right) / 2$ and $\left(q^{2}-1\right) / 2$, respectively, and $\bar{\omega}_{1}=\theta_{3}+\theta_{7}$ and $\bar{\omega}_{2}=\theta_{4}+\theta_{8}$ are the two distinct Weil characters of degree $q^{2}$. Then

$$
\begin{gathered}
\left(\theta_{3}+\theta_{7}\right) \cdot \chi_{[-,-, 3]}=\theta_{8}+\Phi_{4}+\sum_{k=1}^{t} \xi_{21}^{\prime}(k)+\sum_{k=1}^{t-1} \xi_{42}^{\prime}(k), \\
\left(\theta_{3}+\theta_{7}\right) \cdot \chi_{[-, 2,1]}=\theta_{4}+\Phi_{7}+\Phi_{9}+\sum_{k=1}^{t} \xi_{22}(k)+\sum_{k=1}^{t-1} \xi_{41}(k),
\end{gathered}
$$


TABLE 2. Restrictions of unipotent characters of $\operatorname{Sp}_{6}(q)$

\begin{tabular}{|c|c|c|c|c|c|}
\hline \multirow[t]{3}{*}{$\Lambda$} & \multicolumn{5}{|c|}{$\operatorname{Res}_{P}^{G}\left(\chi_{\Lambda}\right)$} \\
\hline & \multirow[t]{2}{*}{ Type 1} & \multicolumn{3}{|c|}{ Type 2} & \multirow[t]{2}{*}{ Type 3} \\
\hline & & Type 1 & Type 2 & Type 3 & \\
\hline $\begin{array}{c}{[2,1,1]} \\
{[-, 3,1]} \\
{[21,-, 1]} \\
{[1,-, 3]} \\
{[1,2,1]} \\
{\left[1^{2}, 1,1\right]} \\
{\left[1,1^{2}, 1\right]} \\
{[-, 21,1]} \\
{\left[1^{3},-, 1\right]} \\
{[-, 1,3]}\end{array}$ & $\begin{array}{c}{[2,-, 1]+[1,1,1]} \\
{[-, 2,1]} \\
{[2,-, 1]+\left[1^{2},-, 1\right]} \\
{[-,-, 3]} \\
{[1,1,1]+[-, 2,1]} \\
{\left[1^{2},-, 1\right]+[1,1,1]} \\
{\left[-, 1^{2}, 1\right]+[1,1,1]} \\
{\left[-, 1^{2}, 1\right]+[-, 2,1]} \\
{\left[1^{2},-, 1\right]} \\
{[-,-, 3]}\end{array}$ & $\begin{array}{c}{[-, 1,1]} \\
{[1,-, 1]+[-, 1,1]} \\
{[1,-, 1]+[-, 1,1]} \\
{[-, 1,1]} \\
{[1,-, 1]}\end{array}$ & $\begin{array}{l}1_{P_{2}} \\
1_{P_{2}} \\
1_{P_{2}}\end{array}$ & $\begin{array}{l}\nu_{3} \\
\nu_{4} \\
\nu_{7} \\
\nu_{8}\end{array}$ & $\begin{array}{c}\theta_{3} \\
\theta_{4} \\
\theta_{7} \\
\theta_{8} \\
\Phi_{9} \\
\theta_{2}+\theta_{5} \\
\alpha \vartheta_{[-, 21,1]} \\
\vartheta_{[-, 21,1]} \\
\alpha \vartheta_{[-, 1,3]} \\
\vartheta_{[-, 1,3]}\end{array}$ \\
\hline \multicolumn{6}{|c|}{$\vartheta_{[-, 21,1]}:=\theta_{1}+\theta_{4}+\Phi_{7}+\Phi_{9}+\sum_{1 \leq k \leq \frac{1}{2}(q-1)} \xi_{22}(k)+\sum_{1 \leq k \leq \frac{1}{2}(q-3)} \xi_{41}(k)$} \\
\hline \multicolumn{6}{|c|}{$\vartheta_{[-, 1,3]}:=\theta_{6}+\theta_{8}+\Phi_{4}+\sum_{1 \leq k \leq \frac{1}{2}(q-1)} \xi_{21}^{\prime}(k)+\sum_{1 \leq k \leq \frac{1}{2}(q-3)} \xi_{42}^{\prime}(k)$} \\
\hline
\end{tabular}

$$
\begin{gathered}
\left(\theta_{3}+\theta_{7}\right) \cdot \chi_{[1,1,1]}=\theta_{2}+\theta_{3}+\theta_{5}+\Phi_{8}+\Phi_{9}+\sum_{k=1}^{t} \xi_{21}(k)+\sum_{k=1}^{t-1} \xi_{42}(k) \\
\left(\theta_{3}+\theta_{7}\right) \cdot \chi_{\left[1^{2},-, 1\right]}=\theta_{2}+\theta_{5}+\theta_{7}+\Phi_{3}+\sum_{k=1}^{t} \xi_{22}^{\prime}(k)+\sum_{k=1}^{t-1} \xi_{41}^{\prime}(k)
\end{gathered}
$$

Proof. Using the character table of $\operatorname{Sp}_{4}(q)$ given in [10] together with the corrections in [9], one can verify the claimed decompositions of $\left(\theta_{3}+\theta_{7}\right) \cdot \chi_{\Lambda}$ for $\Lambda \in\left\{[-,-, 3],[-, 2,1],[1,1,1],\left[1^{2},-, 1\right]\right\}$, except for the following three typos (in the notation of [10]): $\Phi_{3}\left(C_{21}(i)\right)=$ $-\tilde{\varepsilon}^{\prime}, \xi_{41}^{\prime}(k)\left(D_{1}\right)=\frac{1}{2}\left(q^{2}-1\right)\left((-1)^{k}-(-1)^{t}\right)=\frac{1}{2}\left(q^{2}-1\right) d(k, t)$, and $\Phi_{7}\left(B_{3}(i, j)\right)=(-1)^{i}+(-1)^{j}=s(i, j)$. These corrections can be found in $[13]$. 


\section{The Restrictions of the unipotent Characters of $\operatorname{Sp}_{6}(q)$}

Now let $n=6$, so that $G=\operatorname{Sp}_{6}(q), q$ odd, and $L=A \times L^{\prime}$ with $L^{\prime}=\operatorname{Sp}_{4}(q)$.

For a symbol $\Lambda$, if $\sigma$ is the unipotent character of $L$ labelled by $\Lambda$, then we denote by ${ }^{1} \psi_{\Lambda}$ the character ${ }^{1} \psi_{\sigma}$ of $P$.

Theorem 6.1. Let $\chi=\chi_{\Lambda}$ be a non-trivial unipotent character of $G=\operatorname{Sp}_{6}(q)$ different from the Steinberg character. Then $\operatorname{Res}_{P}^{G}\left(\chi_{\Lambda}\right)$ is as given in Table 2. In particular, $\operatorname{Res}_{P}^{G}\left(\chi_{\Lambda}\right)$ is multiplicity free.

Proof. Since the unipotent characters of $G$ have pairwise different degrees, they are $\alpha$-invariant and Lemma 4.2 can be applied. The Type 1 components of the restrictions are easily determined by HarishChandra theory. The values of the unipotent characters on the class $C_{1}$ containing $\mathbf{z}_{1}$ (see the introduction to Section 4) can be found in [8] or CHEVIE [4].

Case 1: $\Lambda=[21,-, 1]$ and $[2,1,1]$.

We have

$$
R_{L}^{G}\left(1_{L}\right)=\chi_{[3,-, 1]}+\chi_{[21,-, 1]}+\chi_{[2,1,1]} .
$$

Thus Corollary 3.5 yields for the sum of the Type 2 and the Type 3 components of $\operatorname{Res}_{P}^{G}\left(\chi_{[21,-, 1]}+\chi_{[2,1,1]}\right)$ :

$$
2 \cdot{ }^{2} \psi_{1_{P_{4}}}+{ }^{3} \psi_{\bar{\omega}_{1}}^{1,+}+{ }^{3} \psi_{\bar{\omega}_{2}}^{2,+}=2 \cdot{ }^{2} \psi_{1_{P_{4}}}+{ }^{3} \tilde{\psi}_{\theta_{7}}^{+}+{ }^{3} \tilde{\psi}_{\theta_{3}}^{+} .
$$

Let $\chi:=\chi_{\Lambda}$ with $\Lambda=[21,-, 1]$. Then

$$
\chi(1)=\frac{1}{2} q(q+1)\left(q^{3}+1\right) \quad \text { and } \quad \chi\left(\mathbf{z}_{1}\right)=\frac{1}{2} q(q+1)\left(q^{2}+1\right) .
$$

We have ${ }^{1} \chi={ }^{1} \psi_{1_{L}}+{ }^{1} \psi_{\left[1^{2},-, 1\right]}$ and so, by Lemma 4.2,

$$
{ }^{3} \chi(1)=\frac{1}{2} q^{2}(q-1)\left(q^{2}-1\right) \text { and } \quad{ }^{2} \chi(1)=q^{4}-1 .
$$

Since ${ }^{2} \psi_{\mu}(1)=\left(q^{4}-1\right) \mu(1)$, it follows from (13) that $\mu=1_{P_{4}}$. Note that $1_{P_{4}}$, being a Type 1 character of $P_{4}$, gives the entry $[1,-, 1]$ in the corresponding row and column of Table 2 . In the same way we obtain ${ }^{3} \chi$. Then (13) also gives the restriction of $\chi_{[2,1,1]}$.

Case 2: $\Lambda=[-, 3,1],[-, 21,1]$ or $[1,2,1]$.

We have

$$
R_{L}^{G}\left(\chi_{[-, 2,1]}\right)=\chi_{[-, 3,1]}+\chi_{[-, 21,1]}+\chi_{[1,2,1]} .
$$


By (3) and Theorem 3.3 we have

$$
\begin{aligned}
\operatorname{Res}_{P}^{G}\left(\chi_{[-, 3,1]}+\chi_{[-, 21,1]}+\chi_{[1,2,1]}\right)= & { }^{1} \psi_{\chi_{[-, 2,1]}} \\
& \left.+\operatorname{Ind}_{R Q_{K}}^{P}{ }^{s} \operatorname{Res}_{R Q_{K}}^{P}\left(\chi_{[-, 2,1]}\right)\right) \\
& +\operatorname{Ind}_{L}^{P}\left(\chi_{[-, 2,1]}\right) .
\end{aligned}
$$

By Theorem 5.3 we obtain

$$
\operatorname{Res}_{P_{4}}^{L^{\prime}}\left(\chi_{[-, 2,1]}\right)={ }^{1} \psi_{\chi_{[-, 1,1]}}+{ }^{3} \tilde{\psi}_{\nu_{4}}^{+} .
$$

Theorem 3.3(d) and 3.3(e) yield for the sum of the Type 2 and the Type 3 contribution of (14):

$$
{ }^{2} \psi_{[-, 1,1]}+{ }^{3} \tilde{\psi}_{\vartheta}^{+}
$$

with

$$
\vartheta=R_{L_{4}}^{L^{\prime}}\left(1_{A_{4}}^{-} \otimes \nu_{4}\right)=\Phi_{9}+\theta_{1}+\theta_{4} .
$$

(see Lemma 5.1(b)).

Finally, Theorem 3.3(a) and Remark 3.4 give

$$
\operatorname{Ind}_{L}^{P}\left(\chi_{[-, 2,1]}\right)={ }^{1} \psi_{[-, 2,1]}+{ }^{2} \psi_{[-, 1,1]}+{ }^{2} \psi_{\mu}+{ }^{3} \tilde{\psi}_{\vartheta}^{+},
$$

with $\mu={ }^{3} \tilde{\psi}_{\nu_{4}}^{+}$and $\vartheta=\left(\theta_{7}+\theta_{3}\right) \cdot \chi_{[-, 2,1]}$.

Let $\chi=\chi_{\Lambda}$ with $\Lambda=[-, 3,1]$. Then

$$
\chi(1)=\frac{1}{2} q\left(q^{2}+1\right)\left(q^{2}-q+1\right) \quad \text { and } \quad \chi\left(\mathbf{z}_{1}\right)=-\frac{1}{2} q(q-1)\left(q^{2}+1\right) .
$$

From ${ }^{1} \chi={ }^{1} \psi_{[-, 2,1]}$ and Lemma 4.2 we obtain

$$
{ }^{3} \chi(1)=\frac{1}{2} q^{2}(q-1)\left(q^{2}+1\right) \text { and } \quad{ }^{2} \chi(1)=0 .
$$

Thus ${ }^{3} \chi={ }^{3} \tilde{\psi}_{\vartheta}^{+}$with a character $\vartheta$ of $\operatorname{Sp}_{4}(q)$ of degree $\left(q^{2}+1\right) / 2$. On the other hand, by (16) and (17), the irreducible constituents of $\vartheta$ are among the constituents of $\Phi_{9}+\theta_{1}+\theta_{4}$ or $\left(\theta_{7}+\theta_{3}\right) \cdot \chi_{[-, 2,1]}$. Proposition 5.4 now implies that $\vartheta=\theta_{4}$.

Next let $\chi=\chi_{\Lambda}$ with $\Lambda=[1,2,1]$. Then

$$
{ }^{1} \chi={ }^{1} \psi_{[1,1,1]}+{ }^{1} \psi_{[-, 2,1]} .
$$

In addition,

$$
\chi(1)=q^{2}\left(q^{2}+q+1\right)\left(q^{2}-q+1\right) \quad \text { and } \quad \chi\left(\mathbf{z}_{1}\right)=q^{2} .
$$

By Lemma 4.2,

$$
{ }^{3} \chi(1)=q^{3}(q-1)\left(q^{2}+1\right) \quad \text { and } \quad{ }^{2} \chi(1)=q\left(q^{4}-1\right) .
$$


Since ${ }^{2} \psi_{\mu}(1)=\left(q^{4}-1\right) \mu(1)$, it follows that

$$
{ }^{2} \chi={ }^{2} \psi_{\mu}
$$

with $\mu$ a character of $P_{4}$ of degree $q$. By (15) and (17), this implies that $\mu=\chi_{[-, 1,1]}$.

Also, ${ }^{3} \chi$ is labelled by a character $\vartheta$ of $\operatorname{Sp}_{4}(q)$ of degree $q\left(q^{2}+1\right)$. Proposition 5.4 now implies as above that $\vartheta=\Phi_{9}$.

The result for $\chi_{[-, 21,1]}$ now also follows.

Case 3: $\Lambda=\left[1^{3},-, 1\right]$ and $\left[1^{2}, 1,1\right]$.

We have

$$
R_{L}^{G}\left(\chi_{\left[1^{2},-, 1\right]}\right)=\chi_{\left[1^{3},-, 1\right]}+\chi_{[21,-, 1]}+\chi_{\left[1^{2}, 1,1\right]} .
$$

By (3) and Theorem 3.3 we have

$$
\begin{aligned}
\operatorname{Res}_{P}^{G}\left(\chi_{\left[1^{3},-, 1\right]}+\chi_{[21,-, 1]}+\chi_{\left[1^{2}, 1,1\right]}\right)= & \psi_{\chi_{\left[1^{2},-, 1\right]}} \\
& +\operatorname{Ind}_{R Q_{K}}^{P}{ }^{s}\left(\operatorname{Res}_{R Q_{K}}^{P}\left(\chi_{\left[1^{2},-, 1\right]}\right)\right) \\
& +\operatorname{Ind}_{L}^{P}\left(\chi_{\left[1^{2},-, 1\right]}\right) .
\end{aligned}
$$

By Theorem 5.3 we obtain

$$
\operatorname{Res}_{P_{4}}^{L^{\prime}}\left(\chi_{\left[1^{2},-, 1\right]}\right)={ }^{1} \psi_{\chi_{[1,-, 1]}}+{ }^{2} \psi_{1_{P_{2}}}+{ }^{3} \tilde{\psi}_{\nu_{7}}^{+}
$$

Theorem 3.3(d) and 3.3(e) yield for the sum of the Type 2 and the Type 3 contribution of (18):

$$
{ }^{2} \psi_{[1,-, 1]}+{ }^{2} \psi_{\mu}+{ }^{3} \tilde{\psi}_{\vartheta}^{+}
$$

with $\mu=\operatorname{Ind}_{r P_{4} \cap P_{4}}^{P_{4}}\left(1_{P_{2}}\right)$ and $\vartheta=R_{L_{4}}^{L^{\prime}}\left(1_{A_{4}}^{-} \otimes \nu_{7}\right)$. By Theorem 3.3(b) we have

$$
\mu=\operatorname{Ind}_{r P_{4} \cap P_{4}}^{P_{4}}\left(1_{P_{2}}\right)={ }^{1} \psi_{[1,-, 1]}+{ }^{1} \psi_{[-, 1,1]}+{ }^{2} \psi_{1_{P_{2}}},
$$

and, by Lemma 5.1(a),

$$
\vartheta=R_{L_{4}}^{L^{\prime}}\left(1_{A_{4}}^{-} \otimes \nu_{7}\right)=\theta_{5}+\theta_{7}
$$

Finally, Theorem 3.3(a) and Remark 3.4 give

$$
\operatorname{Ind}_{L}^{P}\left(\chi_{\left[1^{2},-, 1\right]}\right)={ }^{1} \psi_{\left[1^{2},-, 1\right]}+{ }^{2} \psi_{[1,-, 1]}+{ }^{2} \psi_{\mu_{1}}+{ }^{2} \psi_{\mu_{2}}+{ }^{3} \tilde{\psi}_{\vartheta}^{+},
$$

with $\mu_{1}={ }^{2} \psi_{1_{P_{2}}}, \mu_{2}={ }^{3} \tilde{\psi}_{\nu_{7}}^{+}$and $\vartheta=\left(\theta_{7}+\theta_{3}\right) \cdot \chi_{\left[1^{2},-, 1\right]}$.

Taking into account that we have already computed the restriction of $\chi_{[21,-, 1]}$, by (19), (20) and (22), the labels for the Type 2 constituents of $\operatorname{Res}_{P}^{G}\left(\chi_{\left[1^{3},-, 1\right]}+\chi_{\left[1^{2}, 1,1\right]}\right)$ are exactly the constituents of

$$
2 \cdot{ }^{1} \psi_{[1,-, 1]}+{ }^{1} \psi_{[-, 1,1]}+2 \cdot{ }^{2} \psi_{1_{P_{2}}}+{ }^{3} \tilde{\psi}_{\nu_{7}}^{+} .
$$


Similarly, by (19), (21) and (22), the labels for the Type 3 constituents of $\operatorname{Res}_{P}^{G}\left(\chi_{\left[1^{3},-, 1\right]}+\chi_{\left[1^{2}, 1,1\right]}\right)$ are exactly the constituents of

$$
\theta_{5}+\left(\theta_{7}+\theta_{3}\right) \cdot \chi_{\left[1^{2},-, 1\right]} \text {. }
$$

Let $\chi=\chi_{\Lambda}$ with $\Lambda=\left[1^{2}, 1,1\right]$. Then

$$
{ }^{1} \chi={ }^{1} \psi_{[1,1,1]}+{ }^{1} \psi_{\left[1^{2},-, 1\right]}
$$

and in addition,

$$
\chi(1)=q^{3}\left(q^{4}+q^{2}+1\right) \quad \text { and } \quad \chi\left(\mathbf{z}_{1}\right)=q^{3}\left(q^{2}+1\right) .
$$

By Lemma 4.2 ,

$$
{ }^{3} \chi(1)=q^{6}(q-1) \quad \text { and } \quad{ }^{2} \chi(1)=q(q+1)\left(q^{4}-1\right) .
$$

Since ${ }^{2} \psi_{\mu}(1)=\left(q^{4}-1\right) \mu(1)$, it follows that

$$
{ }^{2} \chi={ }^{2} \psi_{\mu}
$$

with $\mu$ a character of $P_{4}$ of degree $q(q+1)$. It follows from (23) that $\mu={ }^{1} \psi_{[1,-, 1]}+{ }^{1} \psi_{[-, 1,1]}+{ }^{2} \psi_{1_{P_{2}}}$.

Moreover ${ }^{3} \chi$ is labelled by a character $\vartheta$ of $\operatorname{Sp}_{4}(q)$ with $\vartheta(1)=q^{4}$. Now $\chi$ is also contained in $R_{L}^{G}\left(\chi_{[1,1,1]}\right)$, and so $\operatorname{Res}_{P}^{G}(\chi)$ is a subcharacter of $\operatorname{Res}_{P}^{G}\left(R_{L}^{G}\left(\chi_{[1,1,1]}\right)\right)$. In particular, the Type 3 component of $\operatorname{Res}_{P}^{G}(\chi)$ is contained in (30) below. This implies that $\vartheta=\theta_{2}+\theta_{5}$.

The result for $\chi=\chi_{\Lambda}$ with $\Lambda=\left[1^{3},-, 1\right]$ now also follows.

Case 4: $\Lambda=\left[1,1^{2}, 1\right]$.

We have

$$
R_{L}^{G}\left(\chi_{[1,1,1]}\right)=\chi_{[2,1,1]}+\chi_{\left[1^{2}, 1,1\right]}+\chi_{[1,2,1]}+\chi_{\left[1,1^{2}, 1\right]} .
$$

By (3) and Theorem 3.3 we have

$$
\begin{aligned}
\operatorname{Res}_{P}^{G}\left(R_{L}^{G}\left(\chi_{[1,1,1]}\right)\right)= & { }^{1} \psi_{\chi_{[1,1,1]}} \\
& +\operatorname{Ind}_{R Q_{K}}^{P}\left(\operatorname{Res}_{R Q_{K}}^{P}\left(\chi_{[1,1,1]}\right)\right) \\
& +\operatorname{Ind}_{L}^{P}\left(\chi_{[1,1,1]}\right) .
\end{aligned}
$$

By Theorem 5.3 we obtain

$$
\operatorname{Res}_{P_{4}}^{L^{\prime}}\left(\chi_{[1,1,1]}\right)={ }^{1} \psi_{\chi_{[1,-, 1]}}+{ }^{1} \psi_{\chi_{[-, 1,1]}}+{ }^{2} \psi_{1_{P_{2}}}+{ }^{3} \tilde{\psi}_{\nu_{3}}^{+} .
$$

Theorem 3.3(d) and 3.3(e) yield for the sum of the Type 2 and the Type 3 contribution of (25):

$$
{ }^{2} \psi_{[1,-, 1]}+{ }^{2} \psi_{[-, 1,1]}+{ }^{2} \psi_{\mu}+{ }^{3} \tilde{\psi}_{\vartheta}^{+},
$$


with $\mu=\operatorname{Ind}_{r P_{4} \cap P_{4}}^{P_{4}}\left(1_{P_{2}}\right)$ and $\vartheta=R_{L_{4}}^{L^{\prime}}\left(1_{A_{4}}^{-} \otimes \nu_{3}\right)$. By Theorem 3.3(b) we have

$$
\mu=\operatorname{Ind}_{{ }^{2} P_{4} \cap P_{4}}^{P_{4}}\left(1_{P_{2}}\right)={ }^{1} \mu_{[1,-, 1]}+{ }^{1} \mu_{[-, 1,1]}+{ }^{2} \mu_{1_{P_{2}}},
$$

and, by Lemma 5.1(b),

$$
\vartheta=R_{L_{4}}^{L^{\prime}}\left(1_{A_{4}}^{-} \otimes \nu_{3}\right)=\Phi_{9}+\theta_{2}+\theta_{3} .
$$

Finally, Theorem 3.3(a) and Remark 3.4 give

$$
\operatorname{Ind}_{L}^{P}\left(\chi_{[1,1,1]}\right)={ }^{1} \psi_{[1,1,1]}+{ }^{2} \psi_{[1,-, 1]}+{ }^{2} \psi_{[-, 1,1]}+{ }^{2} \psi_{\mu_{1}}+{ }^{2} \psi_{\mu_{2}}+{ }^{3} \tilde{\psi}_{\vartheta}^{+}
$$

with $\mu_{1}={ }^{2} \psi_{1_{P_{2}}}, \mu_{2}={ }^{3} \tilde{\psi}_{\nu_{3}}^{+}$and $\vartheta=\left(\theta_{7}+\theta_{3}\right) \cdot \chi_{[1,1,1]}$.

It follows that the Type 3 component of $\operatorname{Res}_{P}^{G}\left(R_{L}^{G}\left(\chi_{[1,1,1]}\right)\right)$ is labelled by the character

$$
\Phi_{9}+\theta_{2}+\theta_{3}+\left(\theta_{7}+\theta_{3}\right) \cdot \chi_{[1,1,1]}
$$

of $\mathrm{Sp}_{4}(q)$.

Taking into account that we have already computed the restrictions of $\chi_{[2,1,1]}, \chi_{\left[1^{2}, 1,1\right]}$ and $\chi_{[1,2,1]}$, the labels for the Type 2 constituents of $\operatorname{Res}_{P}^{G}\left(\chi_{\left[1,1^{2}, 1\right]}\right)$ are exactly the constituents of

$$
{ }^{1} \psi_{[1,-, 1]}+{ }^{1} \psi_{[-, 1,1]}+{ }^{2} \psi_{1_{P_{2}}}+{ }^{3} \tilde{\psi}_{\nu_{3}}^{+}
$$

Similarly, the labels for the Type 3 constituents of $\operatorname{Res}_{P}^{G}\left(\chi_{\left[1,1^{2}, 1\right]}\right)$ are exactly the constituents constituents of $\left(\theta_{7}+\theta_{3}\right) \cdot \chi_{[1,1,1]}-\theta_{5}$. The result for $\chi_{\left[1,1^{2}, 1\right]}$ follows.

Case 5: $\Lambda=[1,-, 3]$ or $[-, 1,3]$.

We have

$$
R_{L}^{G}\left(\chi_{[-,-, 3]}\right)=\chi_{[1,-, 3]}+\chi_{[-, 1,3]} .
$$

By (3) and Theorem 3.3 we obtain

$$
\begin{aligned}
\operatorname{Res}_{P}^{G}\left(\chi_{[1,-, 3]}+\chi_{[-, 1,3]}\right)= & \chi_{[-,-, 3]} \\
& +\operatorname{Ind}_{R Q_{K}}^{P}\left(\operatorname{Res}_{R Q_{K}}^{P}\left(\chi_{[-,-, 3]}\right)\right) \\
& +\operatorname{Ind}_{L}^{P}\left(\chi_{[-,-, 3]}\right) .
\end{aligned}
$$

From Theorem 5.3 we have

$$
\operatorname{Res}_{P_{4}}^{L^{\prime}}\left(\chi_{[-,-, 3]}\right)={ }^{3} \tilde{\psi}_{\nu_{8}}^{+} .
$$

Thus Theorem 3.3(e) yields

$$
\operatorname{Ind}_{R Q_{K}}^{P}\left(\operatorname{Res}_{R Q_{K}}^{P}\left(\chi_{[-,-, 3]}\right)\right)={ }^{3} \tilde{\psi}_{\vartheta}^{+},
$$

with $\vartheta=R_{L_{4}}^{L^{\prime}}\left(1_{A_{4}}^{-} \otimes \nu_{8}\right)$. By Lemma 5.1(a),

$$
\vartheta=R_{L_{4}}^{L^{\prime}}\left(1_{A_{4}}^{-} \otimes \nu_{8}\right)=\theta_{6}+\theta_{8} .
$$


Finally, Theorem 3.3(a) and [1, Proposition 2.3] give

$$
\operatorname{Ind}_{L}^{P}\left(\chi_{[-,-, 3]}\right)={ }^{1} \psi_{[-,-, 3]}+{ }^{2} \psi_{\mu}+{ }^{3} \tilde{\psi}_{\vartheta}^{+},
$$

with $\mu={ }^{3} \tilde{\psi}_{\nu_{8}}^{+}$and $\vartheta=\left(\theta_{7}+\theta_{3}\right) \cdot \chi_{[-,-, 3]}$.

Now let $\chi=\chi_{\Lambda}$ with $\Lambda=[1,-, 3]$. Then

$$
\chi(1)=\frac{1}{2} q(q-1)\left(q^{3}-1\right) \quad \text { and } \quad \chi\left(\mathbf{z}_{1}\right)=-\frac{1}{2} q(q-1)\left(q^{2}+1\right) .
$$

Harish-Chandra theory gives

$$
{ }^{1} \chi={ }^{1} \psi_{[-,-, 3]} .
$$

By Lemma 4.2,

$$
{ }^{3} \chi(1)=\frac{1}{2} q^{2}(q-1)\left(q^{2}-1\right) \quad \text { and } \quad{ }^{2} \chi(1)=0 .
$$

Hence

$$
{ }^{3} \chi={ }^{3} \tilde{\psi}_{\vartheta}^{+}
$$

for some character $\vartheta$ of $L^{\prime}$ of degree $\left(q^{2}-1\right) / 2$. Proposition 5.4 and (34) now imply that $\vartheta=\theta_{8}$. This also concludes the proof for $\chi_{\Lambda}$ with $\Lambda=[-, 1,3]$.

\section{ACKNOWLEDGEMENTS}

We thank Frank Lübeck for clarifying discussions on the subtleties of the parametrization of unipotent characters as well as for his hint to reference [11].

Most of this work was done during two visits of the second author at the Department of Mathematics of the University of Auckland in 2007 and 2008. He wishes to express his sincere thanks to all the persons of the department for their hospitality, and also to the Marsden Fund of New Zealand who supported his visits via grant \#9144/3608549.

\section{REFERENCES}

[1] J. An And G. Hiss, Restricting the Steinberg character in finite symplectic groups, J. Group Theory 9 (2006), 251-264.

[2] R.W. Carter, Finite groups of Lie type: Conjugacy classes and complex characters, Wiley, 1985.

[3] C. W. Curtis and I. Reiner, Methods of representation theory Vol. II, Wiley, 1987.

[4] M. Geck, G. Hiss, F. Lübeck, G. Malle, and G. Pfeiffer, CheVie - A system for computing and processing generic character tables, AAECC 7 (1996), 175-210.

[5] P. GÉrardin, Weil representations associated to finite fields, J. Algebra 46 (1977), 54-101. 
[6] G. Hiss and A. Zalesski, appendix by O. Brunat, The Weil-Steinberg character of finite classical groups, Represent. Theory 13 (2009), 427-459.

[7] V. Landazuri and G. M. Seitz, On the minimal degrees of projective representations of the finite Chevalley groups, J. Algebra 32 (1974), 418-443.

[8] F. LüBECK, Charaktertafeln für die Gruppen $\operatorname{CSp}_{6}(q)$ mit ungeradem $q$ und $\operatorname{Sp}_{6}(q)$ mit geradem $q$, Dissertation, University of Heidelberg, 1993.

[9] A. Przygochi, Schur indices of symplectic groups, Comm. Algebra 10 (1982), 279-310.

[10] B. SRInivasan, The characters of the finite symplectic group $S p(4, q)$, Trans. Amer. Math. Soc. 131 (1968), 488-525.

[11] B. Srinivasan, Character sheaves: applications to finite groups. In: Algebraic groups and their generalizations: classical methods (University Park, PA, 1991), pp. 183-194, Proc. Sympos. Pure Math., 56, Part 1, Amer. Math. Soc., Providence, RI, 1994.

[12] P. H. Tiep And A. E. Zalesski, Some characterizations of the Weil representations of the symplectic and unitary groups, J. Algebra 192 (1997), $130-165$.

[13] H. Yamada, The characters of $\operatorname{Sp}_{4}(q), q$ odd, Preprint.

J.A.: Department of Mathematics, University of Auckland, Private Bag 92019, Auckland, New Zealand

G.H.: Lehrstuhl D für Mathematik, RWTH Aachen University, 52056 Aachen, Germany

E-mail address: J.A.: an@math.auckland.ac.nz

E-mail address: G.H.: Gerhard.Hiss@Math.RWTH-Aachen.DE 